\title{
Sweeping Jet Actuator in a Quiescent Environment
}

\author{
Mehti Koklu* and Latunia P. Melton $^{\dagger}$ \\ NASA Langley Research Center, Hampton, VA 23681
}

\begin{abstract}
This study presents a detailed analysis of a sweeping jet (fluidic oscillator) actuator. The sweeping jet actuator promises to be a viable flow control actuator candidate due to its simple, no moving part structure and its high momentum, spatially oscillating flow output. Hot-wire anemometer and particle image velocimetry measurements were carried out with an emphasis on understanding the actuator flow field in a quiescent environment. The time averaged, fluctuating, and instantaneous velocity measurements are provided. A modified actuator concept that incorporates high-speed solenoid valves to control the frequency of oscillation enabled phase averaged measurements of the oscillating jet. These measurements reveal that in a given oscillation cycle, the oscillating jet spends more time on each of the Coanda surfaces. In addition, the modified actuator generates four different types of flow fields, namely: a non oscillating downward jet, a non oscillating upward jet, a non oscillating straight jet, and an oscillating jet. The switching from an upward jet to a downward jet is accomplished by providing a single pulse from the solenoid valve. Once the flow is switched, the flow stays there until another pulse is received. The oscillating jet is compared with a non oscillating straight jet, which is a typical planar turbulent jet. The results indicate that the oscillating jet has a higher (5 times) spreading rate, more flow entrainment, and higher velocity fluctuations (equal to the mean velocity).
\end{abstract}

\section{Nomenclature}

$\alpha=$ flow angle, degree

$K_{2} \quad=$ pitch factor

$U=$ velocity magnitude, $\mathrm{m} / \mathrm{s}$

Ueff = effective cooling velocity, $\mathrm{m} / \mathrm{s}$

Umax = local maximum velocity, $\mathrm{m} / \mathrm{s}$

$\mathrm{x} \quad=$ streamwise location, $\mathrm{mm}$

$\mathrm{z}=$ spanwise location, $\mathrm{mm}$

Abbreviations:

HWA $=$ hot-wire anemometer

PIV $=$ particle image velocimetry

RMS $=$ root mean square

$S W J \quad=$ sweeping jet

$V G J=$ vortex generator jet

Subscripts:

$\therefore \quad=$ angle, degree

$=$ root mean square of fluctuating value

\section{Introduction}

Active flow control has become a rapidly growing field in applied fluid dynamics because of its potential to dramatically improve the system performance of air vehicles. Active flow control implies energy addition to the flow-field via an actuator to yield large-scale changes in the flow field of interest with minimal energy expenditure. Depending on the application, these changes are targeted to improve system performance through lift enhancement, drag reduction, jet thrust vectoring, mixing enhancement, pressure recovery, and heat transfer enhancement, etc. It

\footnotetext{
* Research Engineer, Flow Physics and Control Branch, MS 170, Member AIAA.

${ }^{\dagger}$ Research Engineer, Flow Physics and Control Branch, MS 170, Senior Member AIAA.
} 
has been shown that unsteady actuation is more beneficial for optimal performance as it requires less energy input while satisfying the output requirements ${ }^{1}$. Common unsteady flow control actuators are: oscillatory blowing valves ${ }^{1}$, pulsed jets ${ }^{2}$, pulsed vortex generating jets ${ }^{3-5}$, plasma actuators ${ }^{6-7}$, synthetic jets ${ }^{8}$, and fluidic nozzles ${ }^{9}$. All of these unsteady actuation mechanisms have strengths and weaknesses. An ideal actuator should be simple, have few moving parts, and function over a range of flow conditions. The fluidic oscillator has the potential to be an ideal candidate actuator for application on an air vehicle because it meets all of the above requirements and also has the following prominent advantages: no moving parts, simple and compact design, maintenance free, dimensional scalability, high frequency and amplitude bandwidths, and chemical and temperature resistance.

Although primarily developed as a fluid amplifier, fluidic oscillators have been used in many diverse applications, such as windshield washer fluid nozzles ${ }^{10}$, flow metering devices ${ }^{11-12}$, shower heads, and hot tubs. Recently, there has been a growing interest in using these oscillators as a flow control actuator. For example, the performance of a single-element high-lift airfoil was increased substantially (by up to a $66 \%$ increase in lift at large flap deflections) by employing a spanwise array of fluidic oscillators ${ }^{13}$. A similar performance increase was also achieved by Seele et al. ${ }^{14}$ using fluidic oscillators where the lift-to-drag ratio of a V-22 wing-nacelle combination was increased by $60 \%$. In addition, these actuators reduced the download force of a powered tilt-rotor model by almost $30 \%$ by eliminating the separation at large flap deflections ${ }^{14}$. It was also shown that these actuators prevented flow separation, increased lift, and decreased the drag of a NACA 0021 airfoil at steady and unsteady flap deflections. These actuators were shown to eliminate dynamic stall effects by a significant gain in lift and a substantial reduction in drag within a short period of time ${ }^{15}$. The fluidic oscillator actuators were successfully applied to wind turbine blades where up to a $60 \%$ lift increase was reported ${ }^{16}$. They have also been used to reduce drag by $20 \%$ on trucks ${ }^{17}$. In a recent study, these actuators were applied on a vertical tail (NACA 0012 form) of a typical twin-engine aircraft at Reynolds numbers up to 1.5 million and a 50-70\% increase in the side force was reported ${ }^{18}$. In most of these studies, an engineering optimization of the actuator parameters was not performed, thus the actuator performance could increase with further optimization. Some of the fluidic oscillator actuation mechanisms use a splitter to divide the oscillating jet into discrete outlets however having a continuous sweeping jet from one side to the other side resulted in a remarkable performance increase for separation control ${ }^{13,16,18}$. Thus the fluidic oscillator that creates an oscillatory sweeping jet from side to side will be referred to as a sweeping jet (SWJ) actuator in this paper.

Despite the encouraging results, there are only a few studies that provide measurements of the oscillating flow field out of these actuators. Most of the studies were focused on the frequency response of these actuators ${ }^{19-21}$. Some flow visualization studies, using water ${ }^{19}$, Schlieren ${ }^{22}$, and pressure sensitive paints ${ }^{21}$ shed light on the flow structure. However these studies were qualitative visualizations rather than quantitative measurements. Gregory et al. ${ }^{23}$ used a hot-film anemometer to measure the velocity profile of a simple form of this actuator at low flow rates. Recently, Tomac et al. ${ }^{24}$ used Particle Image Velocimetry (PIV) to measure the liquid flow inside a feedback free fluidic oscillator focusing on the flow structures inside the actuator. In order to better understand the effects of the SWJ actuators, detailed quantitative measurements of the oscillatory flow field produced by these actuators are needed.

The objective of this study is therefore to provide detailed measurements of the SWJ actuator's oscillating flow field. The oscillating flow field was measured in a quiescent environment and air was used as the working fluid. Both hot-wire anemometer (HWA) and PIV techniques were conducted to provide the time averaged, fluctuating, and instantaneous velocity measurements. Measurements of a non oscillating jet using the same actuator were also made to compare the effects of the oscillating and non oscillating jets. Phase averaged PIV measurements were performed to understand the time evolution of the oscillating flow field. This study provides a comprehensive set of data to improve our understanding of the SWJ actuator's oscillatory flow field.

\section{Experiment Description}

\section{A. Sweeping Jet Actuator}

The SWJ actuator was fabricated from ABS plastics with a CNC machine. Another piece, a cover, was glued and bolted to the actuator to prevent any leaks. The orifice cross section has a square shape (aspect ratio is one) and the width is approximately $6.4 \mathrm{~mm}$ (1/4”). The orifice outer sidewalls are $\pm 50^{\circ}$ from the horizontal plane. Figure 1 illustrates the working principle of the SWJ actuator that has been used previously ${ }^{19,25}$. The general operational principle of the bi-stable fluidic actuator has been known for a long time ${ }^{19}$. In short, fluid passes through the actuator main channel and attaches to either Coanda surface due to the Coanda effect (Fig. 1a). A backflow develops in the feedback loop and forces the jet flow to detach from that surface (Fig. 1b) and attach to the opposite surface (Fig. 1c). When the flow attaches to the opposite surface, then a backflow is developed in the other feedback loop which 
forces the jet to switch back to its initial state (Fig. 1a). The process then repeats itself, thus producing a selfsustaining oscillation.

\section{B. Hot-wire Anemometer}

The velocity measurements at the exit of the SWJ actuator were first conducted using a constant temperature anemometer. The experimental set up for HWA measurements is shown in Fig. 2. The probe is a single sensor platinum-plated tungsten wire with a diameter of $5 \mu \mathrm{m}$ and a length of $1.25 \mathrm{~mm}$. The probe was mounted on a twoaxis computer-controlled translation stage with the wire perpendicular to the actuator plane. The probe was connected to the HWA and then to a data acquisition unit. The voltage signal was filtered with a $10 \mathrm{kHz}$ low-pass filter. The signals from the HWA were sampled at $25.6 \mathrm{kHz}$ and 51,200 data points were recorded at each station. To characterize the flow field, the probe was moved in the $z$ direction and the measurements were acquired at 70 stations for the $x=6 \mathrm{~mm}$ streamwise location. However due to increased jet spreading farther away from the exit (at $x=12 \mathrm{~mm}$ and $18 \mathrm{~mm}$ ), more stations were included in the measurements to capture the entire velocity profile. The hot-wire probe was calibrated before each experimental set using a commercial desktop calibration unit. A fourthorder polynomial curve fit was applied to the calibration data and used to convert the voltage signal to velocity. Since the calibrator and the SWJ actuator used the same air source, the temperature variations were expected to be small. The baseline case is defined such that the mass flow rate is fixed to $4.54 \mathrm{~g} / \mathrm{s}(0.01 \mathrm{lb} / \mathrm{s})$ and the probe is placed at $x=6 \mathrm{~mm}$. The baseline HWA measurements were repeated many times (recalibrated every time) and the scatter (maximum RMS of the error) was calculated to be 1.6\%. Based on the accuracy in the calibration and the scatter in the experiment, the uncertainty in the mean velocity measurements was estimated to be less than $2 \%$ for velocities between $5 \mathrm{~m} / \mathrm{s}-120 \mathrm{~m} / \mathrm{s}$.

In this current setup, the mass flow rate of the fluid flow that goes into the actuator was monitored with a computer controlled flow meter. The mass flow rate of the flow meter was fixed at $4.54 \mathrm{~g} / \mathrm{s}$. The static pressure near the plenum entrance was also monitored using a differential pressure gauge; however since the pressure inside the actuator was affected by the actuator and experimental configurations, the mass flow rate was chosen as a variable instead of the pressure. The flow temperature and the mass flow rate inside the flow meter were also recorded.

In addition to the highly fluctuating flow field, variations in the flow direction add some complexity in reducing the hot-wire measurements due to the directional response of the hot-wire probes, namely the pitch and yaw responses. For a flow that oscillates in the $x-z$ plane, we can assume insignificant tangential flow to the hot wire and hence the yaw response is negligible. According to Adrian et al. ${ }^{26}$, the pitch response is strictly a result of the flow that is interacting with the hot-wire prongs and support. The voltage output from the HWA is directly related to the heat removed from the hot wire, and the cooling of the hot wire is affected by many parameters including the pitch response that is not negligible for highly oscillating flow fields. When a single-wire hot-wire probe is calibrated, only the velocity magnitude is varied and the direction of the flow is assumed constant and usually parallel to the probe axis. However, if the flow direction deviates from that of the calibrator, the HWA output voltage, hence the velocity reading also deviates from the true velocity. Assuming no tangential flow component, the effective cooling velocity according to Ref. 26 can be expressed as follows

$$
U_{\text {eff }}^{2}=U^{2}\left(\cos ^{2} \alpha+K_{2}^{2} \sin ^{2} \alpha\right)
$$

Here, $\alpha$ is the angle between the flow direction and the probe axis, $K_{2}$ is the pitch factor, and $U$ is the true normal velocity. The pitch factor is usually determined during the calibration by changing the relative angle between the probe and the calibrator. The pitch factor was found to be 1.22 by using a "yaw/pitch modulator" for the current experimental setup and its variation with respect to velocity magnitude was negligible. More information on the directional sensitivity of hot wires can be found in Refs. 26-28. While 10 to 20 degrees of error in the angle results in less than $2 \%$ error in the velocity measurement, the error can go up to $15 \%$ for larger angles. From the geometry of the actuator, we can deduce that the oscillating flow has constantly varying angles between $\pm 50^{\circ}$. At these large flow angles, the pitch response of the probe should be taken into account. The definition of the angle and the effect of this correction are shown in Fig.3. The flow, which will be explained later, is a jet inclined at an angle of approximately $-50^{\circ}$ from the horizontal plane. As seen in this figure, the uncorrected measurements, which represent the effective cooling velocity, are about 15\% more than the PIV measurements. Applying the pitch response correction with a $-50^{\circ}$ angle assumption improved the results greatly.

Another ambiguity comes from the definition of the angle. Since the jet flow oscillates between two exit sidewalls, the flow angle also changes at the rate of the oscillation frequency and it is unknown at a specific time. With a $25.6 \mathrm{kHz}$ sample rate and $225 \mathrm{~Hz}$ actuator oscillation frequency, the hot-wire probe senses the incoming flow from all directions. Since there is no defined angle, an approximation to the flow angle is required. A simple 
approximation to the angle between the flow direction and the probe axis can be made by using the vertical and the horizontal locations of the probe, that is $\alpha=\tan ^{-1}(Z / x)$. However this definition represents the angle between the hot-wire probe axis and the jet centerline. Obviously, this approximation is only valid when the jet centerline hits the probe wire. When the jet centerline is away from the wire, the error in the approximation is at a maximum while the velocity sensed by the probe is at a minimum. For instance, at the extreme situation where the jet centerline is farthest away from the probe, the velocity magnitude is approximately $3 \mathrm{~m} / \mathrm{s}$. The maximum error is $0.45 \mathrm{~m} / \mathrm{s}(15 \%$ of $3 \mathrm{~m} / \mathrm{s}$ ) which is negligible (1.1\%) compared to the mean velocity (approximately $40 \mathrm{~m} / \mathrm{s}$ for a typical case). Therefore this approximation to the angle definition is reasonable.

\section{Particle Image Velocimetry}

The PIV system was set up to measure the horizontal and vertical velocity components of the oscillating flow produced by the SWJ actuator. To create a quiescent environment with a stable particle seeding, the actuator was placed inside the text section of the NASA Langley 15-Inch Wind Tunnel with the tunnel off. The actuator was mounted at the center of the wind tunnel (both in $\mathrm{y}$ and $\mathrm{z}$ directions). The experimental set up for the PIV measurements is given in Fig.4. The mass flow rate of the actuator was controlled by the flow meter and held constant during the experiment. An in-house smoke generator was inserted between the actuator and the flow meter. To eliminate any particle condensation, the smoke generator was inserted at the closest possible location before the actuator plenum. The smoke generator uses polyethylene glycol as the smoke fluid (specific gravity at 1.022) to produce smoke particles. The smoke generator was designed to be used in pressurized environments. It utilizes the same incoming flow to carry the smoke particles hence it does not change the mass flow rate of the actuator. The particle size of this smoke generator was analyzed with a particle analyzer and the mean diameter was found to be 0.8-1.3 $\mu \mathrm{m}$. Using simple Stokes drag analysis ${ }^{29}, 1 \mu \mathrm{m}$ smoke particles have approximately $3 \mathrm{~ms}$ relaxation time. Therefore we can assume that the particles follow the oscillatory flow with negligible lag. The PIV system includes a 1024x1280 CCD camera installed with a $105 \mathrm{~mm}$ Macro lens. The measurement field of view was approximately $90 \mathrm{~mm} \times 75 \mathrm{~mm}$. The camera was placed approximately $1 \mathrm{~m}$ away from the wind tunnel sidewall, perpendicular to the field of view. A double pulse Nd-Yag laser was operated at $15 \mathrm{~Hz}$ and $200 \mathrm{~mJ}$ output per pulse. The light sheet thickness was about $1 \mathrm{~mm}$. The laser light sheet was projected parallel to the actuator mid-section plane $(\mathrm{y}=0$ plane). Laser pulse separations (time between double exposure) were adjusted to $2 \mu \mathrm{s}$, which gave approximately 3.5 pixel displacement for a baseline case where the maximum instantaneous velocity is $120 \mathrm{~m} / \mathrm{s}$. The other essential component in the PIV system was the delay generator. Due to the fact that the flow oscillates at a dominant frequency, the PIV measurements from successively acquired images would be biased toward a dominant phase. Since it is not feasible to acquire the same number of samples as with the HWA measurement, one way to eliminate this problem is to acquire images randomly. That is, the time between two consecutive image pairs is not fixed but random. This was accomplished by using a delay generator and a simple code to generate uniformly distributed random delays that were added to the triggering signal. The flow field was recorded as 2000 randomly sampled instantaneous velocity fields. The sampling error using 2000 image pairs and 100\% fluctuations is approximately $2.3 \%{ }^{29}$. An interrogation window size of $32 \times 32$ pixels with $50 \%$ overlap was used. The image pairs were then processed using a commercial PIV cross-correlation algorithm ${ }^{30}$ to give instantaneous velocity fields and then averaged to give the mean velocity fields.

\section{Phase Averaged PIV Measurements}

Phase averaged PIV measures the instantaneous velocity field at a prescribed phase of the flow oscillation. This measurement is important because it captures the evolution of the flow oscillation over time. Since the oscillations of this type of actuator are solely due to internal fluid dynamics, variations in the flow conditions such as, internal flow separations, variations in the mass flow rate and pressure, and turbulent fluctuations, contribute to the variations in the actuator oscillation. Because of these fluid dynamic related variations, the oscillation frequency of the actuator is not fixed but changes randomly. Even though the oscillations have a dominant frequency, the instantaneous frequency of the actuator can deviate by 10 to $20 \%$. This "jittering" makes it difficult to obtain a phase averaged measurement of the oscillating flow. In order to overcome this problem, the actuator used in this investigation was modified so that the oscillations could be controlled externally by providing periodic pulses to the main jet with an electric input signal, thus mimicking the operation of the original actuator. To accomplish this, two feedback loops on either side of the actuator main channel were replaced with ports. Without these feedback loops, there is no feedback mechanism to generate oscillations. Therefore the flow is a non oscillating turbulent jet that is inclined due to the Coanda effect. A commercially available high-speed solenoid valve was then used to pulse the air supply to each of these ports thus providing the actuation needed to detach the flow from one Coanda surface and attach it to the opposite Coanda surface. The solenoid valve utilizes a piston that seats into a 0.010 -inch orifice, and 
is cycled from a fully open to a fully closed condition. This enables the valve to generate a pulsed flow with variable frequency, duty cycle, and velocity magnitude. Since the pulses were only used to disturb the main flow, the mass flow reduction due to the mechanics of the piston-spring system at high frequencies is insignificant as long as the flow oscillates. Utilizing a second valve at the opposite port and running it $180^{\circ}$ out of phase with the first valve, the main jet goes back and forth between two Coanda surfaces and hence mimics the original actuator oscillation. The high-speed solenoid valves were controlled by a function generator. The valve inlet was connected between the flow meter and the actuator plenum and shared the same air source; therefore the mass flow rate of the actuator remained constant. The schematic of the modified actuator is given in Fig. 5. This type of modification brings numerous advantages. As quoted by Cattafesta et al. in a recent review paper ${ }^{31}$, "Although these actuators are promising, a major drawback is that the oscillation frequency is directly dependent on the flow rate through the device. It is highly desirable to decouple these two parameters." A decoupled actuator can deliver high mass flow rates without changing the frequency or can deliver high frequency oscillating jets with minimal mass flow rates. Therefore this modification allows: 1) decoupling of the frequency and mass flow rate, 2) on demand oscillations, 3) on demand phase difference, 4) synchronous and asynchronous (and any combination) oscillation of the actuator arrays, 5) use in closed loop feedback control applications which greatly improves the flow control, and 6) controlled oscillation angle by providing an asymmetric (unequal amount) of flow to the ports. For example, the oscillations can be restricted to oscillate between the sidewall and the center instead of between two sidewalls. Notwithstanding all of these advantages, the modification was not intended to replace the original actuator but to obtain the phase information of the oscillating flow field. Therefore this study was only focused on the original actuator and the rest of the advantages were left as future work.

With the actuator frequency well defined, the images could be acquired at a specific time delay by synchronizing the image acquisition and laser with the valve drive signal. The same experimental set up explained in Section II.C was used to acquire the PIV images. Within the PIV image acquisition software, the timing delay was varied in order to acquire images at 18 specific phases of the actuator oscillation, which resulted in the measurement of the velocity field at every $20^{\circ}$ of phase. At each phase, 300 image pairs were acquired which equates to 6000 instantaneous velocity fields over one oscillation cycle. Based on the $30 \%$ maximum flow fluctuations measured by the hot-wire anemometer at each phase, the sampling error using 300 image pairs is approximately $1.7 \%{ }^{29}$. The image pairs for each phase were processed using a commercial PIV cross-correlation algorithm ${ }^{30}$ and then averaged to give the phase-averaged velocity field for that particular phase.

\section{Results and Discussion}

\section{A. HWA Results}

The flow field out of the SWJ actuator was first characterized by performing HWA measurements. While the jet flow goes back and forth between two Coanda surfaces inside the actuator, the resulting flow out of the actuator is a highly fluctuating oscillatory flow. In Fig.6, a typical frequency spectrum of the oscillation is shown for this type of actuator. The corresponding peak frequency is $225 \mathrm{~Hz}$, however higher harmonics are also visible in the spectrum. The dominant frequency is very clear and its amplitude is more than any of the turbulent fluctuations. This frequency corresponds to the baseline case where the mass flow rate was fixed at $4.54 \mathrm{~g} / \mathrm{s}$. The probe was located in front of the actuator orifice at $x=6 \mathrm{~mm}$ and $z=10 \mathrm{~mm}$. Although the amplitude of the spectrum varies with the location of the hot-wire probe, the spectrum profile and its peak value does not change. Figure 7 shows a typical time history of the hot-wire signal at the same location. Because the flow oscillates in the $x-z$ plane, the values for $U$ represent the instantaneous velocity magnitude rather than an individual velocity component. As can be seen, the instantaneous velocities can reach up to $120 \mathrm{~m} / \mathrm{s}$. The region of high velocity indicates when the jet flow directly hits the probe, and the region of low velocity indicates when the jet flow is away from the probe. The waveform demonstrates that the probe senses the high and low velocities roughly equal amounts of time. The fluctuations in the velocity waveform are due to the turbulent fluctuations as well as the flow direction uncertainty as described in Section II.A. Looking at the transition between high and low velocities, we can infer that the jet spends a short time in this region and rapidly moves away from the probe. This rapid movement of the jet flow is the consequence of the rapid movement of flow inside the actuator from one Coanda surface to the other Coanda surface. The low velocities that are sensed by the probe while the jet is on the opposite side are due to flow entrainment. However it should be noted that the hot wire was calibrated for $5-120 \mathrm{~m} / \mathrm{s}$ range and outside this range the velocity values have larger errors. Although the velocity magnitude is small $(4 \mathrm{~m} / \mathrm{s})$, the associated error was found to be on the order of $5 \%$ by simply checking the calibrator output velocity with a pitot-static tube for low velocities. The frequency variation of the SWJ actuator was tested by gradually increasing the mass flow rate of the flow meter and recording the peak frequency of the spectrum. Consistent with the data available in the literature, the frequency of the actuator varies 
almost linearly with the mass flow rate; however the oscillation frequency is much lower than other actuators of this type which are described in the literature, as having oscillation frequencies in the $\mathrm{kHz}$ range. The lower frequency of our actuator is due to the fact that the scale is larger and the actuator frequency depends not only on the mass flow rate but also on the geometry of the actuator. There is a slight decline in the frequency response after $250 \mathrm{~Hz}$. This decline is more evident for higher mass flow rates due to the flow choking inside the actuator.

Figure 9 shows the velocity profiles of the SWJ actuator that were measured by HWA. The probe was traversed first in $z$ and then in $x$ directions for a fixed mass flow rate $(4.54 \mathrm{~g} / \mathrm{s})$. To compare the actuator oscillating flow output, a velocity profile of a non oscillating straight jet is also provided. The non oscillating jet was produced using the same actuator however with slight modification as described in Section II.D. To obtain a straight non oscillating jet, high-speed solenoid valves were fully opened and a constant flow was allowed to pass through the valves and then to each port. The steady flow from these ports eliminates the flow attachment to the Coanda surfaces. Therefore neither jet oscillations nor inclination occur but a straight planar turbulent jet is formed. This non oscillating jet is a typical planar turbulent jet that is described in the literature ${ }^{31-33}$. It has a peak velocity at the centerline and very narrow jet width. The oscillating flow out of the SWJ actuators produced completely different velocity profiles. Having the same mass flow rate, using similar geometry and with the probe located at the same location as the non oscillating jet, the maximum velocity of the oscillating flow was decreased almost one third. The velocity profile has double peaks and there is a velocity minimum at the center. This double peak velocity profile is consistent with the actuator physics. When the flow attaches to one of the Coanda surfaces inside the actuator, the flow at the exit attaches to opposite sidewall as well (Fig.1). The flow stays at that side until the feedback flow is strong enough to detach the flow from the Coanda surface. The movement from one side to another is rapid so the time spent in transition is short. Since the mean velocities were obtained by averaging the instantaneous velocities over time, a relatively short time results in smaller values in the mean profile. Therefore even though the mass flow rate provided to the actuator was constant over time, we observed smaller velocity magnitudes at the center. In reverse, since the flow spends more time at each side, the corresponding higher velocity magnitudes in the mean velocity results in double peaks.

Another significant difference is noticed by examining the jet spreading. Usually the jet half width, where the local velocity is half of the maximum velocity, is used for describing the jet spreading. For the non oscillating jet, the jet half width is $8.5 \mathrm{~mm}$ at the $6 \mathrm{~mm}$ streamwise station. For the oscillating jet, it jumps to $40.7 \mathrm{~mm}$ at the same location which shows almost 5 times more spreading than the non oscillating jet. The jet half widths at the $12 \mathrm{~mm}$ and $18 \mathrm{~mm}$ streamwise locations are $55.4 \mathrm{~mm}$ and $69.9 \mathrm{~mm}$ respectively for the oscillation jet. These half widths indicate a $140^{\circ}$ spreading angle while the typical planar turbulent jets have only about an $11^{\circ}$ spreading angle ${ }^{31-33}$.

Figure 10 shows the nondimensional fluctuating velocity profiles. The RMS (root mean square) velocity data obtained from the HWA were nondimensionalized with the local maximum velocity at each downstream location. Again for comparison, the fluctuating velocity profile for the non oscillating jet is also presented in the figure. The fluctuating velocity profile for a non oscillating jet is similar to the turbulent planar jets that are available in the literature ${ }^{31-33}$. Due to the shear flow, there are two peaks in the profile and the profile shows a maximum turbulent intensity of $25-30 \%$. The actuator's highly fluctuating flow field is apparent in this figure which shows $100 \%$ nondimensional fluctuations at the same streamwise location $(x=6 \mathrm{~mm})$. The maximum intensity of the fluctuations slowly decays to $97 \%$ and $90 \%$ for the $\mathrm{x}=12 \mathrm{~mm}$ and $18 \mathrm{~mm}$ streamwise locations, respectively.

Behavior of the oscillating flow field at different mass flow rates was tested by varying the input mass flow rate of the flow meter to 2.27 and $6.81 \mathrm{~g} / \mathrm{s}$. As can be expected, the mean velocity increases as we increase the mass flow rate. The maximum mean velocities are $19.5,39.5$, and $55.8 \mathrm{~m} / \mathrm{s}$ for $2.27,4.54$, and $6.81 \mathrm{~g} / \mathrm{s}$ mass flow rates, respectively. Both the nondimensional mean and the fluctuating velocity profiles do not show a significant difference, except a slight increase between two peaks as the mass flow increases (Figs.11-12). This can be explained by considering the relative time spent between two peaks. As mentioned before, the data between two peaks represent the velocity profile when the jet moves from one side to the other, i.e., transition. As shown in Fig.8, the operating frequency increases almost linearly with the mass flow rate. As the frequency increases, the ratio of the transition time and the dwell time on the Coanda surface increases as well. Since the mean velocity represents the time average, a relatively increased transition time results in an increase in the velocity profiles between two peaks. The slight increase between two peaks results in shrinkage of the profiles outside of these peaks due to the constant mass flow rate.

\section{B. PIV Results}

In a complementary manner to the hot-wire measurements, we also performed PIV measurements at the center

plane of the actuator exit. The PIV measurements provide both instantaneous and mean velocity fields. In Fig.13, an instantaneous 2 dimensional velocity field at the exit of actuator is shown. The maximum flow velocities are on the 
order of $100 \mathrm{~m} / \mathrm{s}$. While the jet flow moves in the angular direction, it also moves in the radial direction. When moving in the radial direction, a pressure pulse is created in front of the jet and this pulse will move forward until the flow starts to detach from the Coanda surface. A gust-like flow at the downside indicates that flow is still moving upward. These structures cannot be observed in the mean velocity measurements (both HWA and PIV) as the averaging smears them out. These flow structures are similar to wind gusts, in that they have localized accelerated flows. The local accelerated flows create many vortices in the flow domain and these vortices travel downstream. These vortices may also contribute to the superiority of these actuators over the other types of active flow control methods.

When the actuator jet flow is fully attached to the actuator exit sidewall, it is very similar to the vortex generating jets (VGJ), which produce streamwise vortices using air jets. Due to the oscillations, the jet flow goes on and off (i.e., pulsates) at each exit sidewall. Therefore the SWJ actuator resembles the pulsed VGJs, which were developed to reduce the mass flow requirements of the steady $\mathrm{VGJ}^{3-5}$. The mechanism of the SWJ actuators can be explained as analogous to the pulsed VGJs. In the literature ${ }^{3-5}$, the following advantages are given for the pulsed VGJs: 1) significantly improve the performance of VGJs by reducing the required mass flow rate, 2) affect a significantly larger area compared to the steady VGJ, 3) enhance flow entrainment both in the near and far fields, and 4) when used in a transverse jet, such as on an airfoil in free stream, the created streamwise vortices penetrate much deeper into the turbulent boundary layer and possibly enhance the mixing process. Similar to the VGJs, it is known that the SWJ oscillating jets substantially reduce the required mass flow rate. It is also obvious from the hotwire measurements that the oscillating jet affects a significantly larger area (more than 5 times) compared to the steady jets. A simple integral analysis will show the jet entrainment of the oscillating vs. non oscillating jets. Unfortunately, because of the continuous flow direction change, it was difficult to deduce any flow entrainment information from the hot-wire measurements. On the other hand, the PIV measurements allow us to extract the velocity components. Assuming constant air density, a simple integration of the $x$-component of the velocity along the $y$ direction at the $x=6 \mathrm{~mm}$ streamwise location indicates that the oscillating jet flow entrainment is almost $20 \%$ more than that of non oscillating jet. In addition to these similar pulsing effects, the SWJ has another advantage that is associated with the oscillating (angular movement) phenomena. The created vortices not only travel in the streamwise (radial) direction but also in the spanwise (angular) direction which may significantly enhance the boundary layer mixing. Furthermore, the created gust-like flow structures affect the near field boundary layer when the jet flow is on the other side.

The histogram of the jet angles shows the distribution of the flow directions (Fig.14). The flow direction angle at an instantaneous moment was calculated by using the jet centerline velocity components and then averaging these angle values for 5 consecutive points near the actuator exit. In this histogram, it is evident that most of the angle values are at $\pm 40^{\circ}$. The double peaks at $\pm 40^{\circ}$ confirm that the jet spends more time at each side. The minimum at $0^{\circ}$ indicates that the jet angular velocity is fastest (least amount of data) at the centerline, similar to a simple harmonic oscillation. The time averaged velocity field (Fig.15) indicates that the maximum mean velocity is approximately 40 $\mathrm{m} / \mathrm{s}$ (recall the maximum instantaneous velocity was approximately $100 \mathrm{~m} / \mathrm{s}$ ). The symmetric velocity field in this figure validates that the random image acquisition method enabled the random sampling of the measurements. The spreading of the oscillating flow is more visible in this figure. Consistent with the angle histogram, most of the flow magnitudes are at $\pm 40^{\circ}$. Note that the orifice outer sidewalls are $\pm 50^{\circ}$ from the horizontal plane.

A comparison of the hot wire and the PIV results is given in Fig.16 for the baseline case. Here it should be noted that since the flow has an unsteady flow direction, the HWA measurements show the velocity magnitude rather than a component. Therefore the presented PIV data in this figure also show the velocity magnitude. The agreement of the PIV and hot-wire measurements is very good for the non oscillating jet. However as we look at the oscillating flow case, the hot-wire results are slightly lower than the PIV results between the first peak and the centerline. Except for these regions, the agreement is reasonable. The mismatch is due to the asymmetric profile obtained in the hot-wire measurements. The first peak is slightly lower $(3 \mathrm{~m} / \mathrm{s})$ than the second peak. Even though the experiments were repeated many times to troubleshoot the reason, an asymmetric velocity profile was obtained. The experiments at different dates (to eliminate any temperature difference and calibration errors), different probes (to eliminate any probe malfunctions), different experimental set up (to eliminate the effect of the hot-wire stand), different mass flow rates, etc. all resulted in an asymmetric velocity profile.

Before proceeding to the phase averaged PIV measurements, different flow types that were obtained by the modified actuator are presented. In the original actuator, the feedback loops are the mechanism that initiate and maintain the oscillations by simultaneously delivering pressure pulses to the main jet inside the actuator. Eliminating these feedback loops also eliminates the oscillation mechanism. In our modification we only blocked the middle part of the feedback loops to minimize the geometric mismatch. Due to the Coanda effect, the main jet attaches to either side of the Coanda surfaces inside the actuator. Therefore the output at the actuator exit is a steady, non oscillating 
but an inclined jet. A similar study was also performed in Ref. 25 using a different type of actuator. In that study, even though a non oscillating jet was observed at the exit (both in the measurements and simulation), the jet flow seemed to be straight rather than inclined. It can be concluded that the Coanda effect in Ref. 25 is weaker than the current actuator so that in the absence of the feedback loops flow does not attach to the Coanda surfaces but tends to be straight. The weaker Coanda effect is due to geometric differences of the Coanda surfaces inside the actuator. However in the current actuator, the Coanda effect was strong enough to attach the fluid flow to the surfaces. A PIV measurement of such an inclined jet is given in Fig. 17. This measurement was obtained by ensemble averaging 300 images pairs. The actuator input mass flow rate was again fixed at $4.54 \mathrm{~g} / \mathrm{s}$. As shown in this figure, the steady jet flow was completely attached to the actuator exit sidewall. Maximum velocities are on the order of $100 \mathrm{~m} / \mathrm{s} \mathrm{similar}$ to the instantaneous velocity profiles of the oscillating jet flow (Fig. 13). The switching from upward jet (Fig. 17a) to downward jet (Fig. 17b) was accomplished by providing a single pulse to the opposite port. The single pulse to the opposite port was created by opening and closing the solenoid valve momentarily. This pulse moves the main jet from one Coanda surface to the opposite Coanda surface hence the flow at the exit is switched from an upward jet to a downward jet. One interesting feature is that when the flow is switched using a single pulse to the other side, the flow stays there until another pulse is received. Therefore this configuration may be utilized as a flow sensor too. It is also possible to create various types of vortex generating jets by using different jet angle combinations such as counter-rotating and/or co-rotating vortices and their numerous combinations.

In Fig.18, the PIV measurement of the non oscillating jet is provided. This measurement was obtained by ensemble averaging 500 images pairs. This jet was previously used to make comparisons between the oscillating and the non oscillating jets. The mass flow rate was $4.54 \mathrm{~g} / \mathrm{s}$. Maximum velocity magnitude is on the order of $100 \mathrm{~m} / \mathrm{s}$. The right figure explains the production of the non oscillating jets. As explained before, an equal amount of steady flow was provided to the ports by fully opening the high speed solenoid valves. The flow from each port eliminates the attachment of the main jet to the Coanda surfaces therefore stabilizing the main jet. Without having the flow attachment to the Coanda surface, the flow at the exit is a steady non oscillating jet that is similar to the planar turbulent jets.

\section{Phase Averaged PIV Results}

Phased averaged PIV measurements present a statistically smoothed motion of the oscillating jet flow field over one cycle. The description of the actuator and the experimental set up that enabled the phase averaged PIV measurements were explained in Section II.D. Basically, we removed the feedback loops and attached two high speed solenoid valves to the ports. The input of these valves is connected to the same air source between the flow meter and the plenum in order not to change the actuator mass flow rate. The experimental set up and the modified actuator concept were given previously in Figs. 4-5.

As consistent with previous measurements, the mass flow rate was fixed at $4.54 \mathrm{~g} / \mathrm{s}$ and the same experimental set up was used. The only difference between the original actuator and this modified actuator was the actuator operating frequency. The original actuator dominant frequency at this mass flow rate is $225 \mathrm{~Hz}$. However in the modified actuator the frequency was selected as $105 \mathrm{~Hz}$, which corresponds to the oscillation frequency when the mass flow rate is set to $2.27 \mathrm{~g} / \mathrm{s}$. This difference is due to the limitation of the solenoid valves and the experimental set up. At the frequency of $225 \mathrm{~Hz}$, the solenoid valves do not operate as expected and the flow out of the SWJ actuator does not oscillate. In addition, the solenoid valves heated up quickly at this frequency. There may be some solutions to eliminate this problem such as using a different type of valve or modifying the geometry to allow more mass flow to pass through to the valves. However, due to the time limitations we decided to use a lower frequency. We also tried different frequencies with the same mass flow rate (not shown here), which only changed the velocity profile between two peaks as explained in Section III.A (Figs. 11-12). The phase averaged PIV measurements of the oscillating flow field were obtained for every 20 degree of phase, resulting 18 specific phases. Figure 19 shows phase averaged PIV measurements at 6 different phases. In Fig. 19a, the phase between the image acquisition signal and the valve drive signal is zero. At this phase, we can see that the flow is in the transition region where it is moving from one Coanda surface to the other. The jet flow is detached from the exit sidewall and moving upward. The jet core is almost straight but the rest of the jet is curved. Because of the averaging, all of the small vortical structures are not present in the figure. Fig. 19b shows the flow field at 40 degree of phase that is $380 \mathrm{~ms}$ after the first figure. As you can see, the jet is still in the transition region, and moving upwards to the other Coanda surface. The jet core passed the centerline plane however the tail is still touching the lower boundary. The velocity magnitude of the tail is reduced to $20 \mathrm{~m} / \mathrm{s}$ from $30 \mathrm{~m} / \mathrm{s}$. The curvature of the jet is increased. Fig. 19c illustrates the jet (phase is $80^{\circ}$ ) $760 \mathrm{~ms}$ after the phase $0^{\circ}$ data shown in Fig. 19a. The jet core seems to be attached to the exit sidewall however the tail of the jet is still moving upward. The velocity magnitude of the tail is very small (on the order of $10 \mathrm{~m} / \mathrm{s}$ ). This movement continues until the jet and its tail are completely inclined with the exit side wall 
which will be given in phase $160^{\circ}$ shown in Fig. 19d. This phase is very similar to the non oscillating inclined jet that was given in Fig.17a. The tail is completely in the upward region. The flow structures presented in previous phases were completely diffused in the region. The next two phases (Fig. 19e-d) show similar flow fields however the jet is moving in opposite directions. The flow finally touches the bottom sidewall similar to the non oscillating downward inclined jet given in Fig. 17b.

\section{Conclusion}

The present study provides a detailed analysis of the sweeping jet (SWJ) actuator flow field in a quiescent environment. The oscillating flow field was first investigated by using the hot-wire anemometer (HWA) technique to obtain local mean and fluctuating velocity profiles at various stations and flow rates. Particle image velocimetry (PIV) measurements were acquired to obtain time averaged and instantaneous velocity flow fields at the exit of the SWJ actuator. The modified actuator that incorporates high-speed solenoid valves to control the oscillation enabled phase averaged PIV measurements of the oscillating jet. In addition, local velocity profiles of hot-wire data and PIV measurements were compared.

When measuring an inclined flow with a HWA, the pitch response of the probe should be taken into account. Although the small-medium angles $(\alpha<20)$ result in less than $2 \%$ error, the error can be as high as $15 \%$ when the angle between flow direction and hot-wire probe axis increases. Therefore for the HWA measurements of the SWJ actuator, where the angle varies between $\pm 60^{\circ}$, the pitch corrections should be applied to obtain the true velocity. It has been shown that this correction greatly improves the HWA measurement results. In the case of unsteady angle variations, which is the case for oscillatory flows, the approximated angle definition resulted in reasonable results. Another critical point when measuring this type of actuator (or flow field) is the usage of random image acquisition in PIV measurements. Since this type of flow field has a dominant frequency, the sequential image acquisition may lead to lock in measurements around the dominant phase. For this purpose, we introduced a simple technique which uses a uniformly distributed random number generator and a delay generator. By using random valued delays, we were able to capture the randomly sampled measurements, which greatly improved the results.

We have observed that the oscillating jet flow out of the SWJ actuator spends more time on each Coanda surface than in the transition from surface to surface. This makes the jet profile have double peaks. The movement of the jet flow between the two Coanda surfaces (transition) is very fast. This rapid transition makes the jet profile have a minimum at the center. This observation is visible in the time history of the velocity signal (at a fixed point), and in the velocity profiles obtained with the hot wire. It is also possible to observe this phenomenon in the jet angle histogram and time averaged velocity profiles of the PIV measurements.

We have also observed that the spreading of the oscillating jet is almost 5 times more than that of the non oscillating jet. In addition, the velocity fluctuations are comparable (or equal) to the jet mean velocity where the non oscillating jets (also the typical planar turbulent jets) have maximum velocity fluctuations that are about $30 \%$ of the mean jet velocity. The increased spreading and higher velocity fluctuations may be the reason for these actuators being superior to other types of flow control actuators.

Increasing the mass flow to the actuators yields a higher oscillation frequency as expected. However, increasing the frequency also reveals an interesting phenomenon. As the frequency increases, the ratio of the transition time and the dwell time on the Coanda surface increases as well. This relatively increased transition time results in an increase in the velocity profiles between two peaks, as we increase the mass flow.

We have concluded from the instantaneous velocity measurements that the oscillating flow creates a gusty flow field in the region where there are accelerated local flow fields. This gusty flow field in the tail of the jet flow created many vortices that travel downstream. We have also developed a modified actuator in order to capture the phase averaged PIV measurements of the oscillating jets. This modification consists of removing the feedback loops and inserting two high speed solenoid valves into the ports. Removing the feedback loops created an interesting flow field in which the flow attaches to either one of the Coanda surfaces and results in a non oscillating inclined jet. The switching from one Coanda surface to the other is possible with a single pulse and once the flow switches it stays there until it receives another pulse from the opposite port. Attaching the flow on the second Coanda surface resulted in an inclined jet in the opposite $z$ direction. Introducing a steady mass flow from these ports resulted in a straight non oscillating jet which is very similar to the turbulent planar jets. In addition, by utilizing the high-speed solenoid valves, it is also possible to obtain an oscillating flow field that is similar to the original unmodified actuator. So, using the modified actuator, it is possible to obtain four different types of flow fields, namely: a non oscillating downward jet, a non oscillating upward jet, a non oscillating straight jet, and an oscillating jet. Among the many advantages, the modified actuator allows us to decouple the actuator amplitude (mass flow rate) and 
frequency. Since the frequency depends on the mass flow rate for the unmodified actuator, the decoupling is a significant improvement, enabling the oscillation of the actuator at a desired frequency and/or mass flow rate.

\section{Acknowledgments}

The authors would like to thank the Subsonic Fixed Wing program for funding the research and the following individuals for their support: Chung-Sheng Yao, John Lin, Catherine McGinley, Luther Jenkins, Jerome Harris, and Charlie Debro.

\section{References}

${ }^{1 .}$ Greenblatt, D. and Wygnanski, I. J., "The control of flow separation by periodic excitation," Progress in Aerospace Sciences, Vol. 36, No.7, 2000, pp. 487-545,

${ }^{2}$ Magill, J. C. and McManus, K. R., "Exploring the feasibility of pulsed jet separation control for aircraft configurations," Journal of Aircraft, Vol. 38, No. 1, 2001, pp. 48-56.

${ }^{3 .}$ McManus, K. R., Legner, H. H., and Davis, S. J., "Pulsed Vortex Generator Jets for Active Control of Flow Separation," AIAA paper 94-2218, June 1994.

4.Tilmann, C. P., Langan, K. J., Betterton, J. G., and Wilson, M. J., "Characterization of Pulsed Vortex Generator Jets for Active Flow Control," Presented at the RTO AVT Symposium on Active Control Technology for Enhanced Performance Operation Capabilities of Military Aircraft, Land Vehicles and Sea Vehicles, Germany, May, 2000.

${ }^{5 .}$ Johari, H., and Rixon, G. S. "Effects of pulsing on a vortex generator jet." AIAA Journal, Vol. 41, No. 12, 2003, pp. 23092315.

${ }^{6}$ Roth, J. R., Sherman, D. M., and Wilkinson, S. P., "Electrohydrodynamic flow control with a glow-discharge surface plasma," AIAA Journal, Vol. 38, No. 7, 2000, pp. 1166-1172.

${ }^{7}$ Enloe, C. L., McLaughlin, T. E., VanDyken, R. D., Kachner, K. D., Jumper, E. J., Corke, T. C., Post, M., and Haddad, O., "Mechanisms and Responses of a Single Dielectric Barrier Plasma Actuator: Geometric Effects," AIAA Journal, Vol. 42, No. 3, 2004, pp. 595-604.

${ }^{8}$ Glezer, A. and Amitay, M., "Synthetic jets" Annual Review of Fluid Mechanics, Vol. 34, 2002, pp. 503-529.

${ }^{9}$ Viets H. "Flip-flop jet nozzle". AIAA Journal, Vol. 13, 1975, pp.1375-1379

${ }^{10}$ Stouffer, R. D., "Liquid Oscillator Device", U.S. Patent 4,508,267, Issued April 2, 1985.

${ }^{11}$ Beale, R. B. and Lawler, M. T., "Development of a wall-attachment fluidic oscillator applied to volume flow metering," Flow: Its measurement and control in science and industry. Vol. 1, ISA, 1974, pp. 989-996.

12.Wang, H., Beck, S. B. M., Priestman, G. H., and Boucher, R. F., "Fluidic pressure pulse transmitting flowmeter," Chemical Engineering Research \& Design, Transactions of the Institute of Chemical Engineers, Part A, Vol. 75, No. A4, 1997, pp. 381391.

${ }^{13}$ DeSalvo, M., Whalen, E., and Glezer, A., "High-Lift Enhancement using Fluidic Actuation,” AIAA 2010-0863, Jan., 2010.

${ }^{14}$ Seele, R., Tewes, P., Woszidlo, R., McVeigh, M. A., Lucas, N. J., and Wygnanski, I. J., "Discrete Sweeping Jets as Tools for Improving the Performance of the V-22," Journal of Aircraft, Vol. 46, No. 6, Nov.-Dec. 2009.

${ }^{15}$ Phillips, E., Woszidlo, R., and Wygnanski, I., "The Dynamics of Separation Control on a Rapidly Actuated Flap", AIAA paper 2010-4246, June, 2010.

${ }^{16 .}$ Cerretelli, C., Wuerz, W., and Gharaibah, E., "Unsteady Separation Control on Wind Turbine Blades Using Fluidic Oscillators," AIAA Journal., Vol. 48, No. 7, 2010, pp. 1302-1311.

${ }^{17}$ Seifert, A., Stalnov, O., Sperber, D.,Arwartz, G., Palei, V.,Davis, S., Dayan, I., and Fono, I., "Large Trucks Drag Reduction Using Active Flow Control”, AIAA 2008-0743, January, 2008.

${ }^{18 .}$ Seele R., Graff, E., Gharib, M., Taubert, L., Lin, J., and Wygnanski, I. "Improving Rudder Effectiveness with Sweeping Jet Actuators", AIAA paper 2012-3244, June 2012

${ }^{19}$ Raman, G., and Raghu, S., "Cavity Resonance Suppression Using Miniature Fluidic Oscillators," AIAA Journal, Vol. 42 , No. 12, 2004, pp. 2608-2612.

${ }^{20}$ Gregory, J. W., Sullivan, J. P., Raman, G., and Raghu, S. "Characterization of a micro fluidic oscillator for flow control" AIAA Paper 2004-2692, June 2004.

${ }^{21}$ Gregory, JW, Sullivan, JP, and Raghu, S, "Visualization of Internal Jet Mixing in a Fluidic Oscillator." Proceedings of the 11th International Symposium on Flow Visualization, University of Notre Dame, 2004.

${ }^{22}$ Gregory, JW, Gnanamanickam, EP, Sullivan, JP, and Raghu, S, "Variable-Frequency Fluidic Oscillator Driven by Piezoelectric Devices." AIAA paper 2005-0108, 2005.

${ }^{23 .}$ Gregory, JW, Ruotolo, JC, Byerley, AR, and McLaughlin, TE, "Switching Behavior of a Plasma-Fluidic Actuator." AIAA paper 2007-0785, Jan. 2007.

24.Tomac, M. N., and Gregory, J. W., "Frequency Studies and Scaling Effects of Jet Interaction in a Feedback-Free Fluidic Oscillator." AIAA paper 2012-1248, January 2012.

${ }^{25 .}$ Vatsa, V., Koklu, M and Wygnanski, I., "Numerical Simulation of Fluidic Actuators for Flow Control Applications", AIAA paper 2012-3239, June, 2012.

${ }^{26 .}$ Adrian, R. J., Johnson, R. E., Jones, B. G., Merati, P., and Tung, T.C. "Aerodynamic disturbances of hot-wire probes and directional sensitivity”, Journal of Physics E: Scientific Instruments, Vol. 17, 1984, pp. 62-71 
27. Jorgensen F. E. "Directional sensitivity of wire and fiber-film probes", Disa Information, No 11, 1971, pp. 31-29.

${ }^{28}$ Wagner, T. C., and Kent, J. C. "On the directional sensitivity of hot-wires: a new look at an old phenomenon." Experiments in Fluids, Vol.6, No. 8, 1988, pp. 553-560.

${ }^{29}$ Adrian, R. J., and Westerweel , J., Particle Image Velocimetry, Cambridge University Press, New York, 2011.

${ }^{30 .}$ Scarano, F. and Riethmuller, M., "Advances in Iterative Multigrid PIV Image Processing" Experiments in Fluids Supplemental, Vol.29, 2000, pp.S51-S60.

${ }^{31 .}$ Cattafesta III, L. N., and Sheplak. M., "Actuators for Active Flow Control." Annual Review of Fluid Mechanics, Vol. 43, 2011, pp.247-272

${ }^{32}$ Bradbury, L. J. S. "The structure of a self-preserving turbulent plane jet." Journal of Fluid Mechanics, Vol.23, No. 01, 1965, pp.31-64

${ }^{33}$ Gutmark, E., and Wygnanski. I. "The planar turbulent jet." Journal of Fluid Mechanics, Vol.73, No. 03, 1976, pp.465-495 

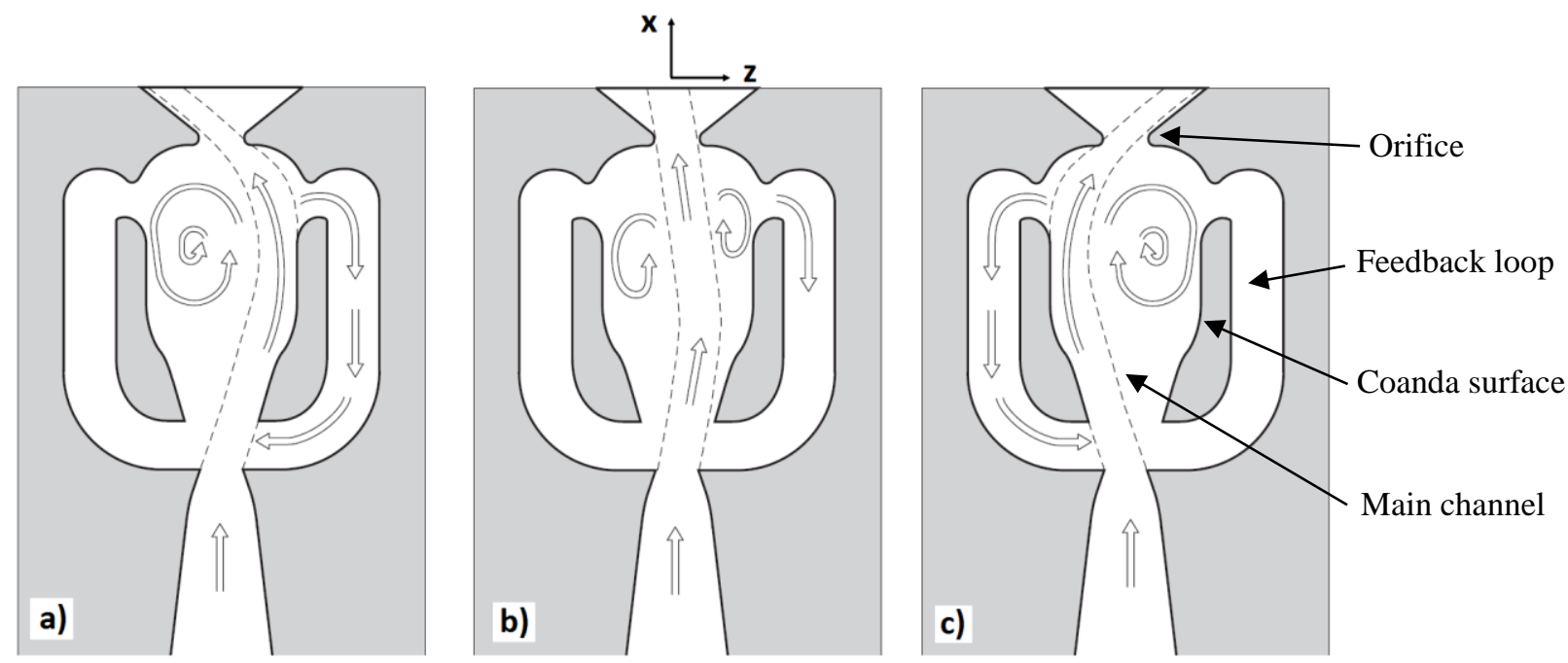

Figure 1. Working principle of the sweeping jet actuator.

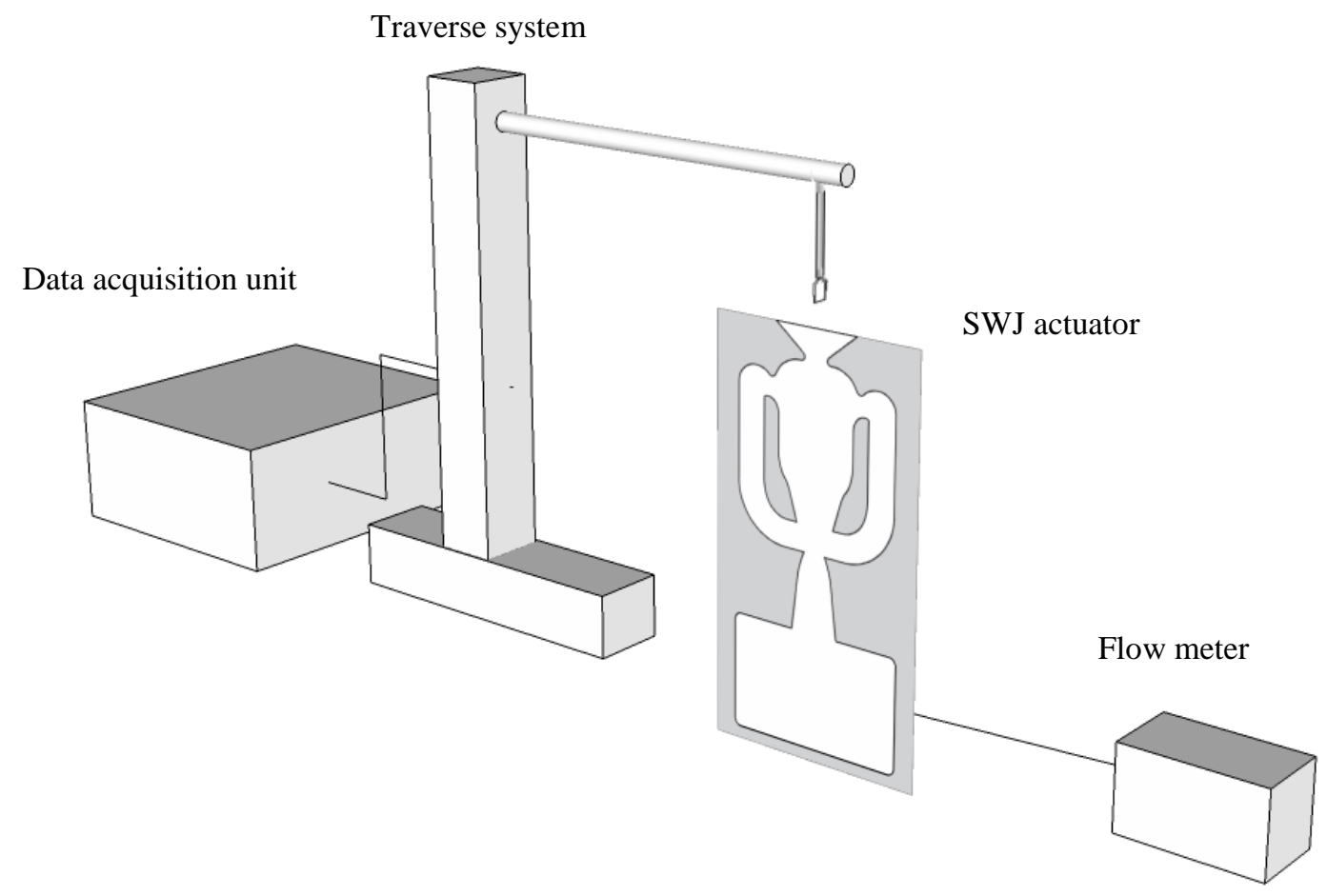

Figure 2. Experimental set up for HWA measurements. 

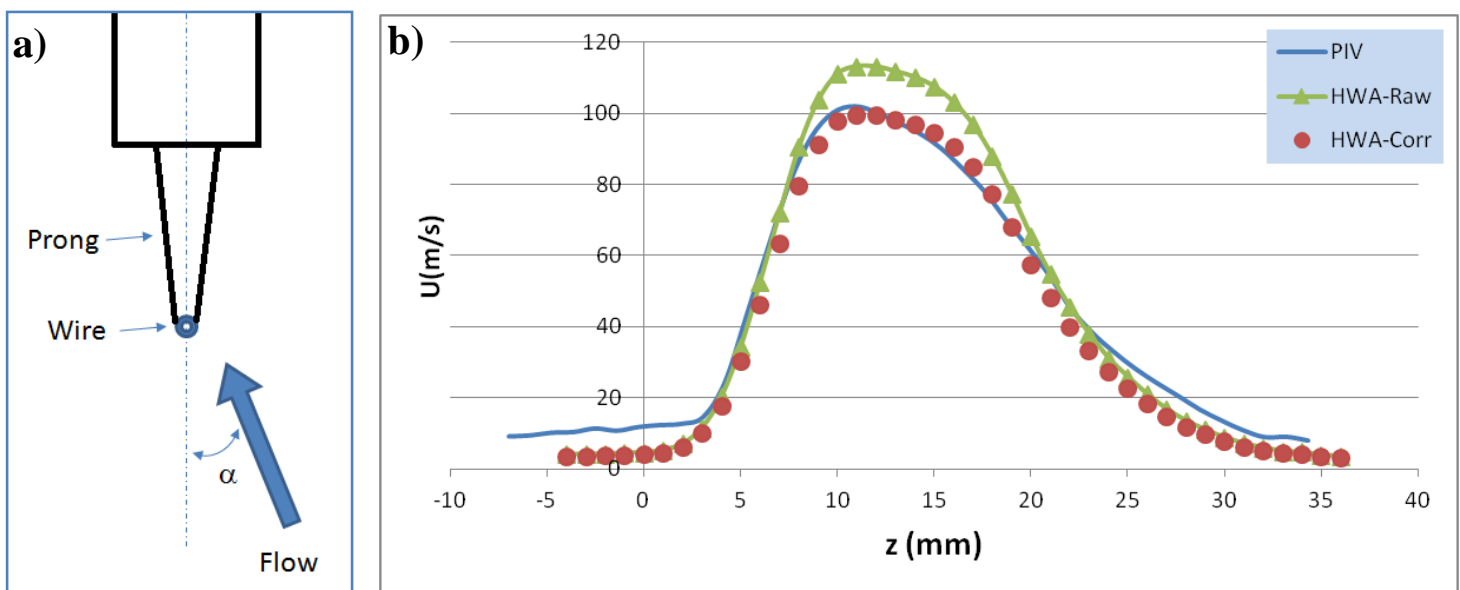

Figure 3. a) Definition of flow angle and b) its corresponding pitch correction.

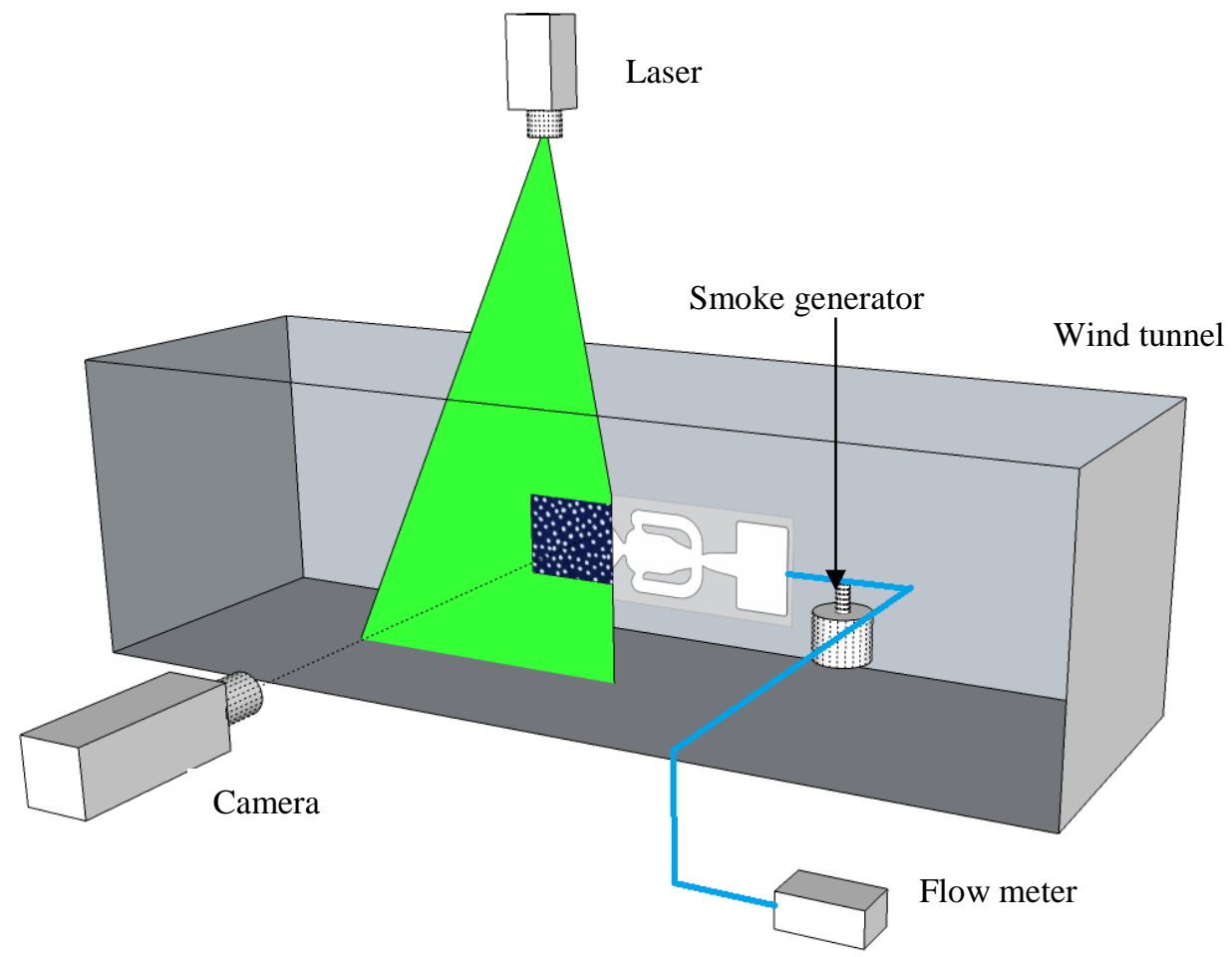

Figure 4. Experimental set up for PIV measurements. 


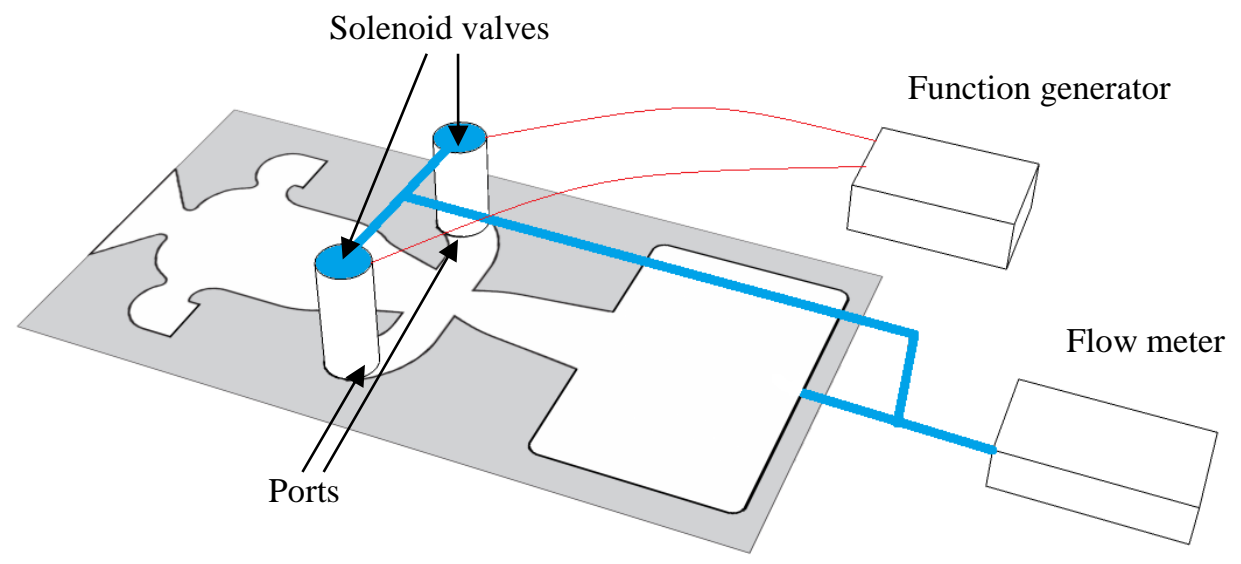

Figure 5. Schematic of the modified actuator.

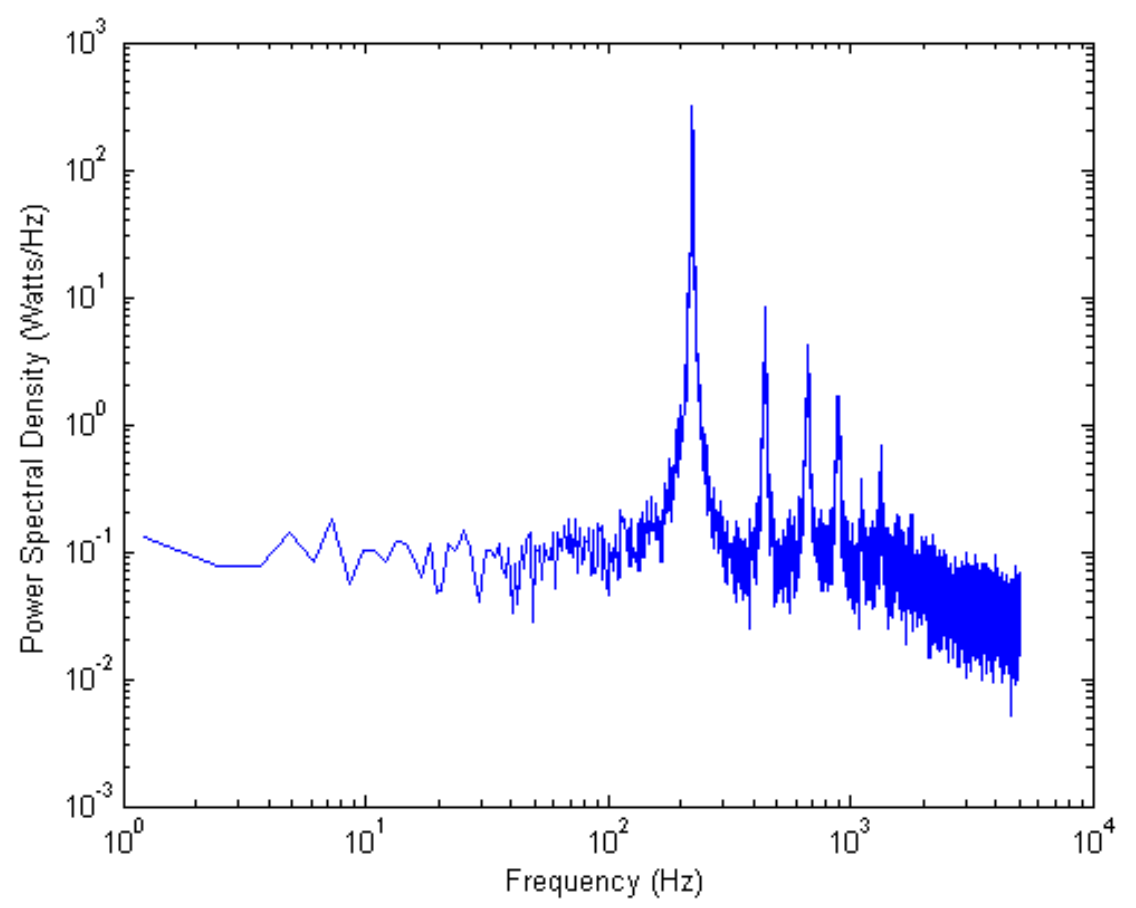

Figure 6. Power spectral density of the frequency response of the actuator for the baseline case 


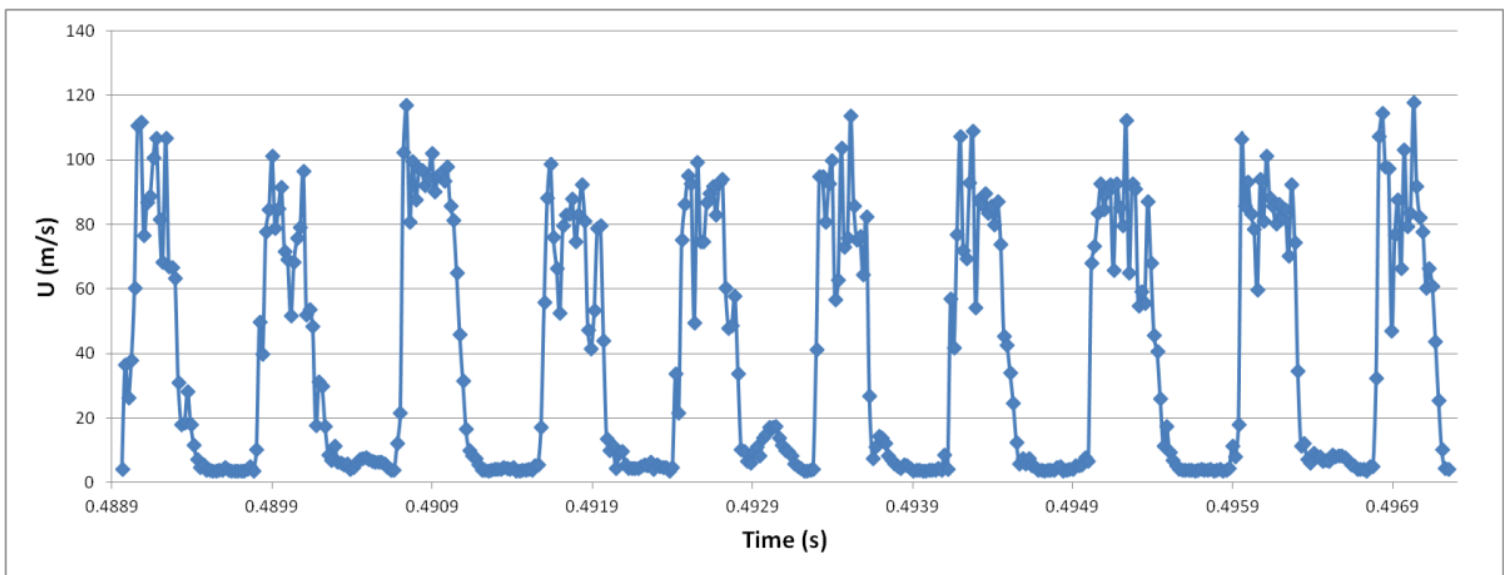

Figure 7. A snapshot of time history of the velocity signal for the baseline case.

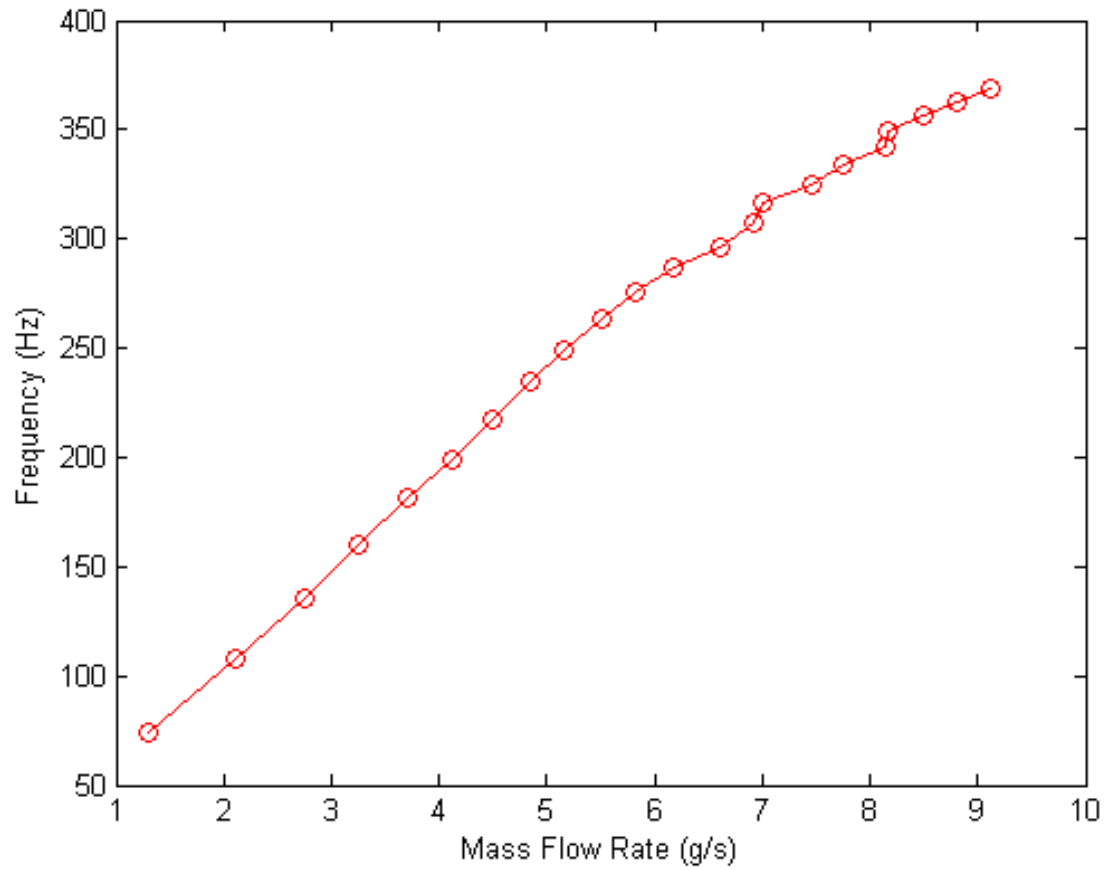

Figure 8. Variation of the actuator frequency with respect to mass flow rate. 


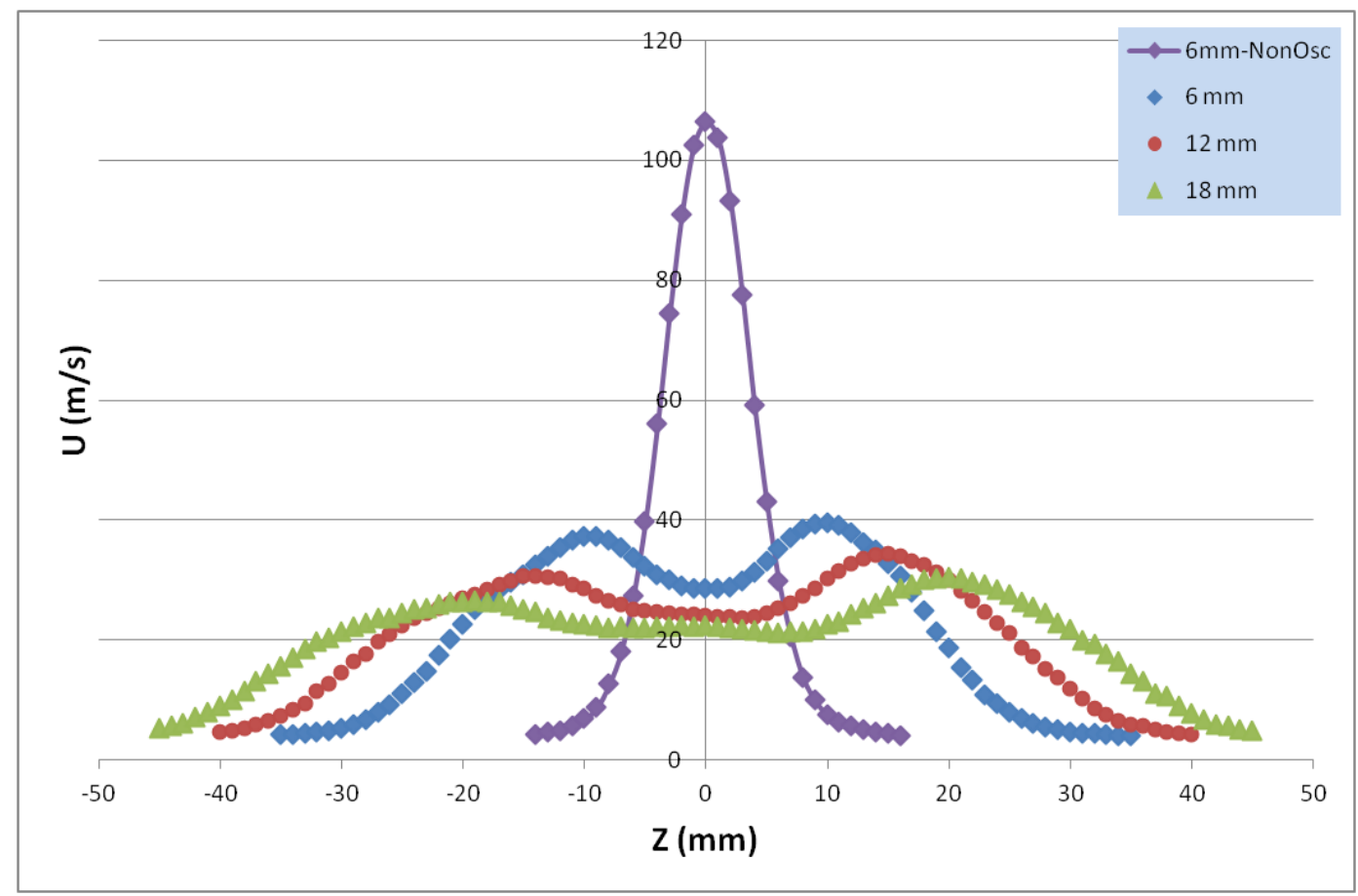

Figure 9. Mean velocity profiles at different $x$ stations (mass flow rate is $4.54 \mathrm{~g} / \mathrm{s}$ )

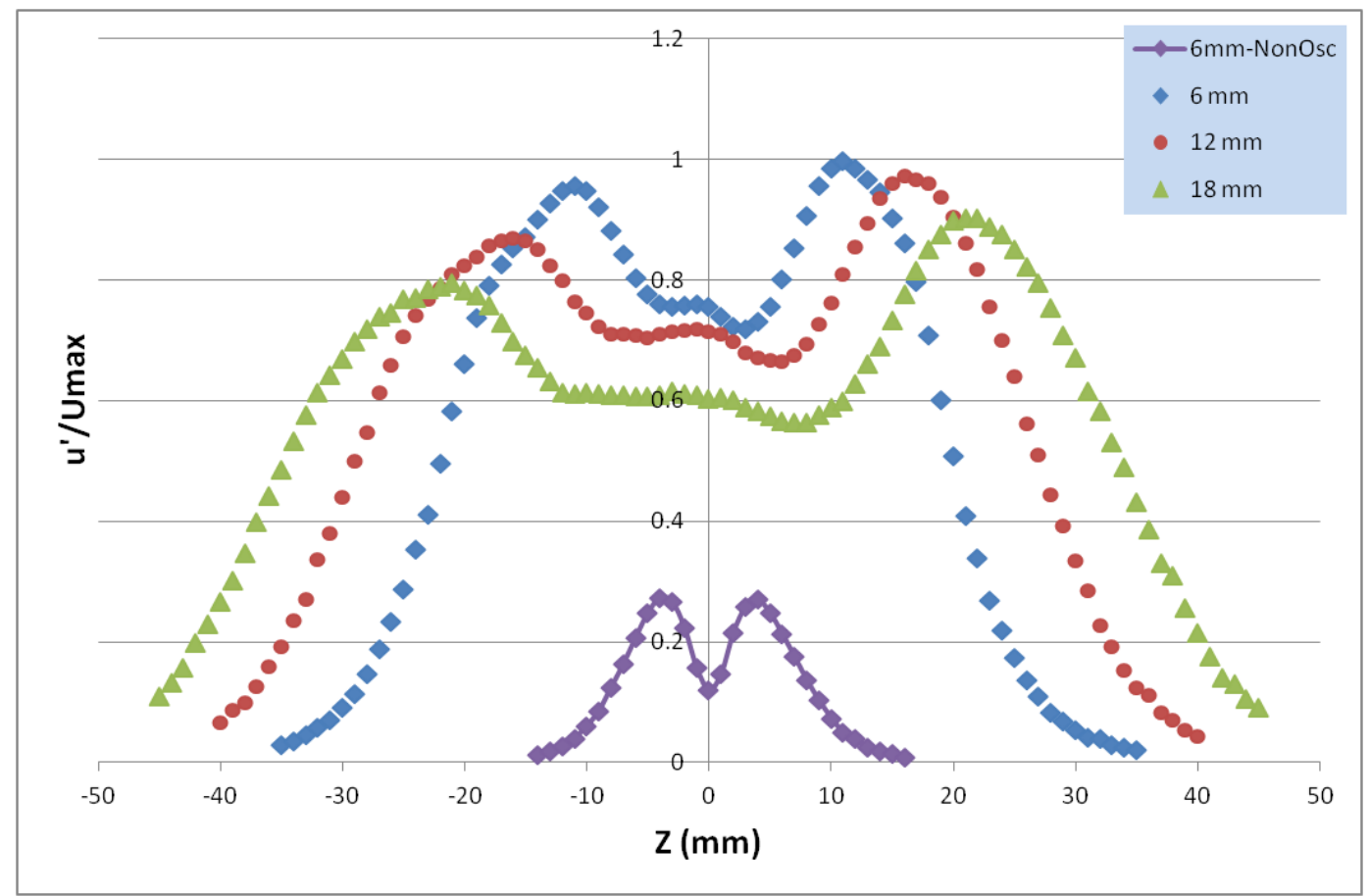

Figure 10. Fluctuating velocity profiles at different $x$ stations (mass flow rate is $4.54 \mathrm{~g} / \mathrm{s}$ ). 


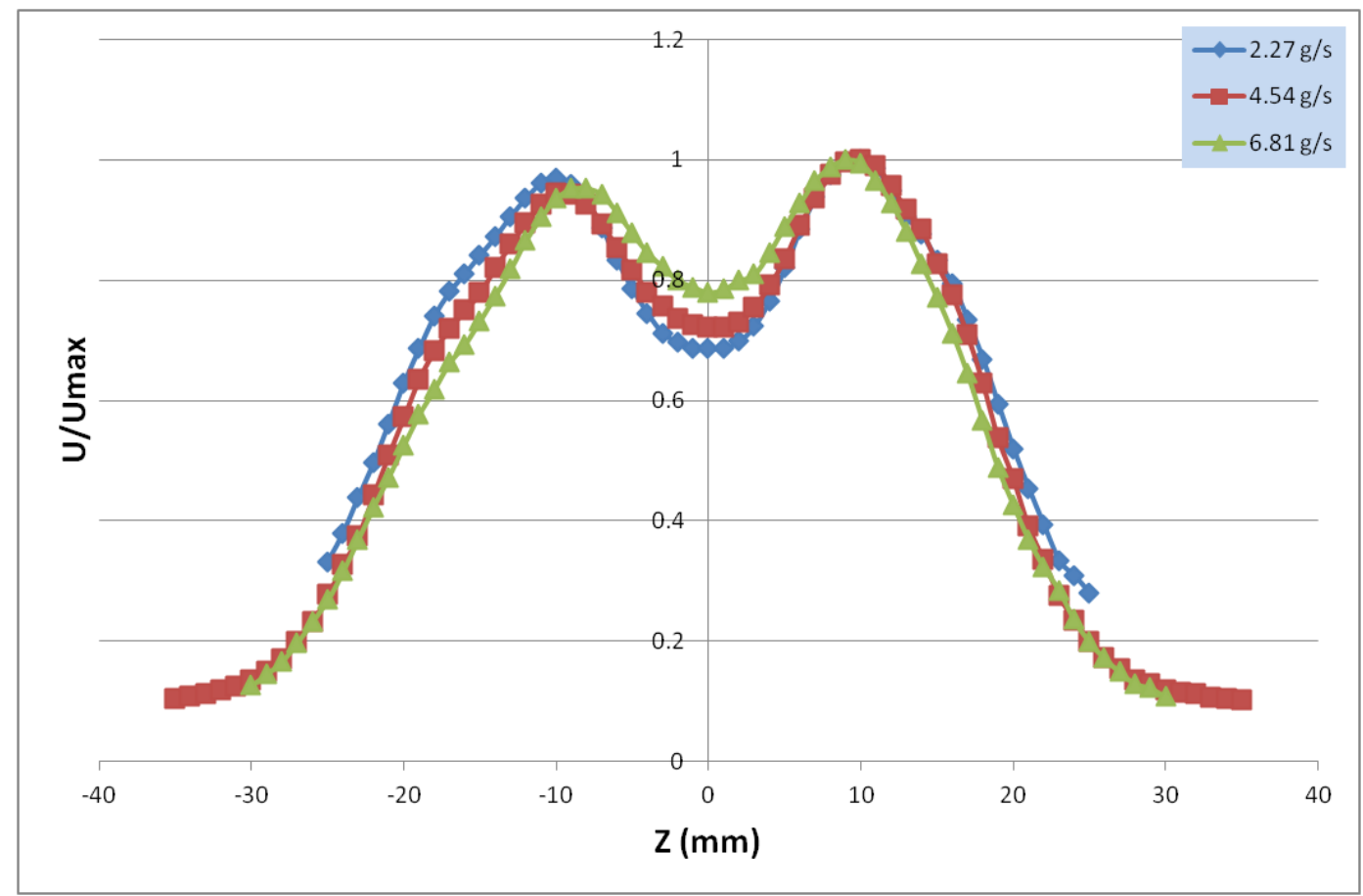

Figure 11. Nondimensional mean velocity profiles at $x=6 \mathrm{~mm}$ at different mass flow rates.

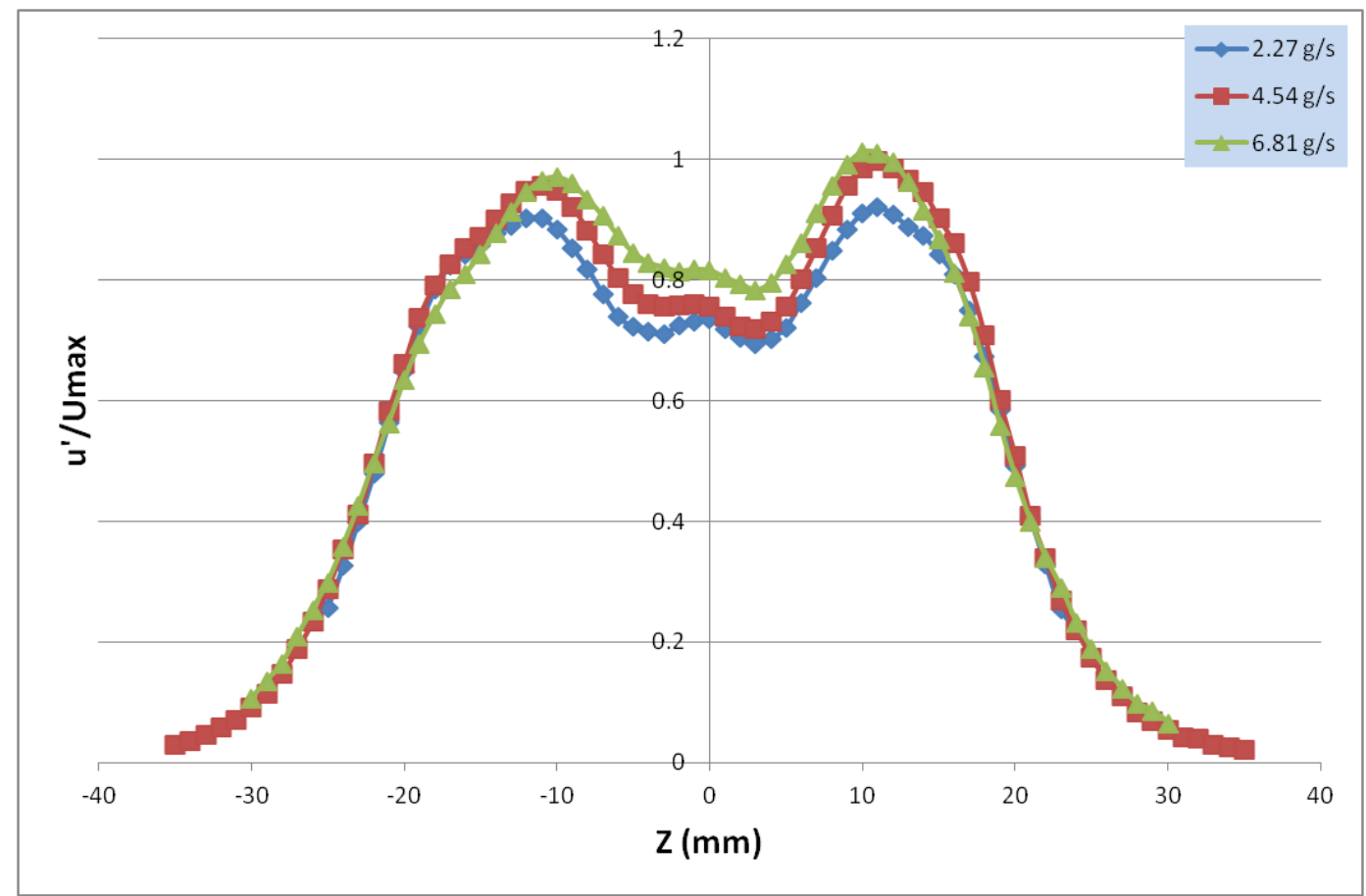

Figure 12. Nondimensional fluctuating velocity profiles at $x=6 \mathrm{~mm}$ at different mass flow rates. 


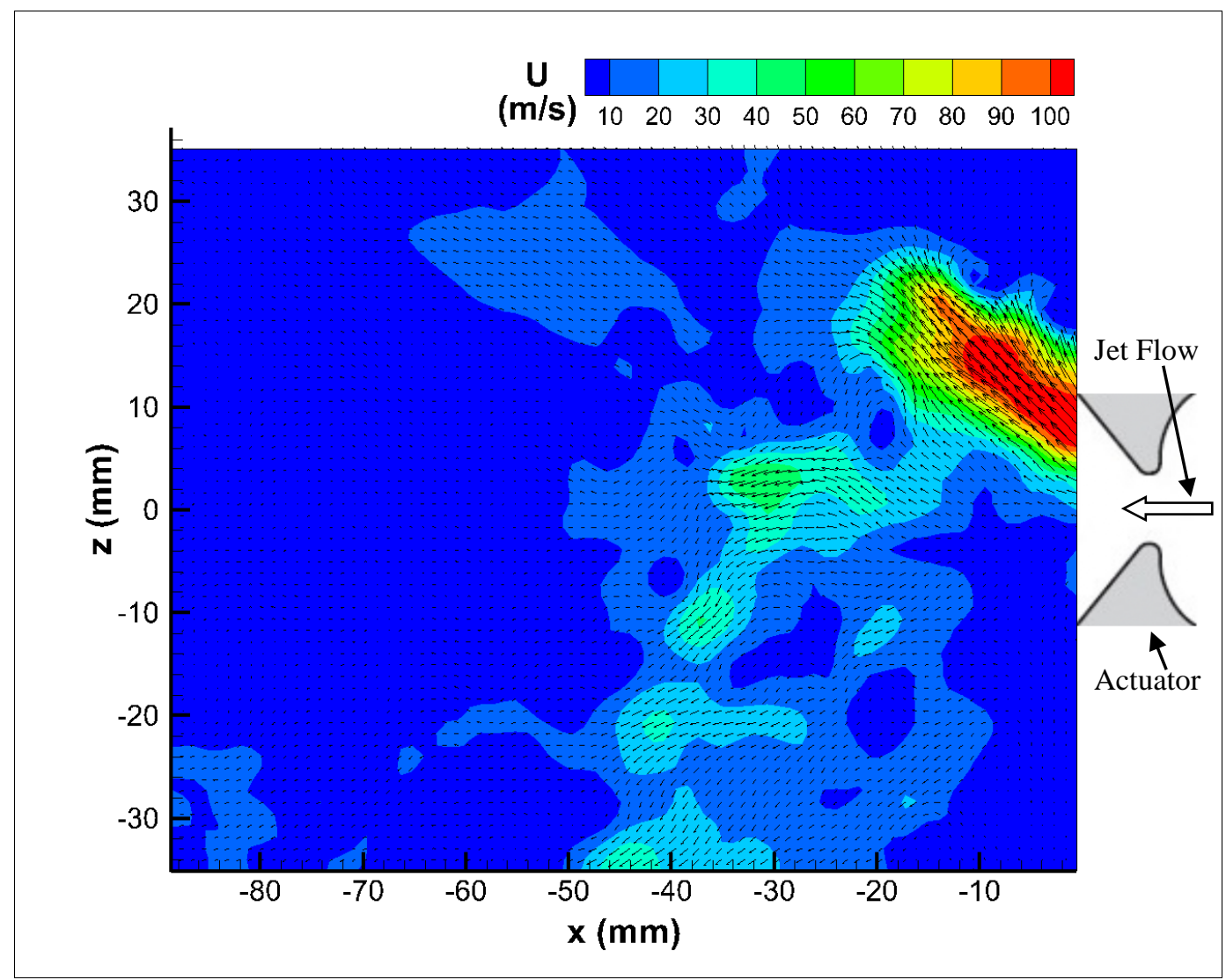

Figure 13. Instantaneous velocity field (mass flow rate is $4.54 \mathrm{~g} / \mathrm{s}$ ).

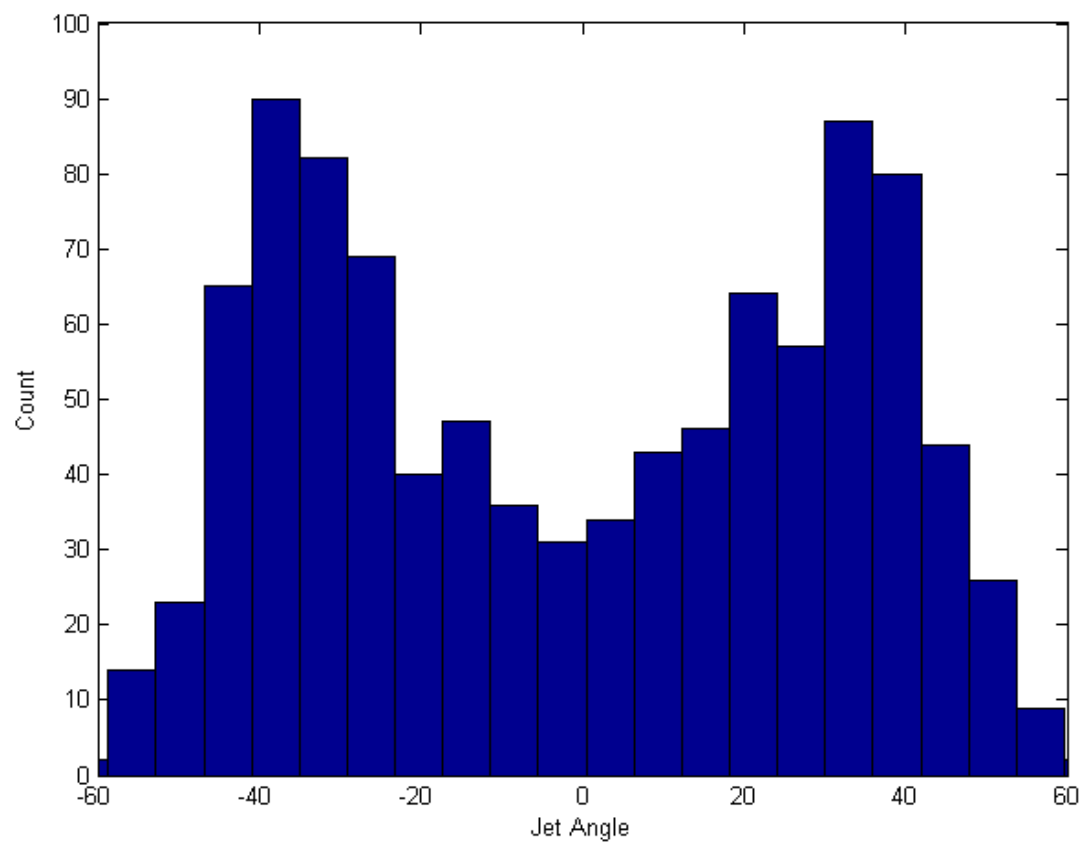

Figure 14. Histogram of the jet angle (mass flow rate is $4.54 \mathrm{~g} / \mathrm{s}$ ). 


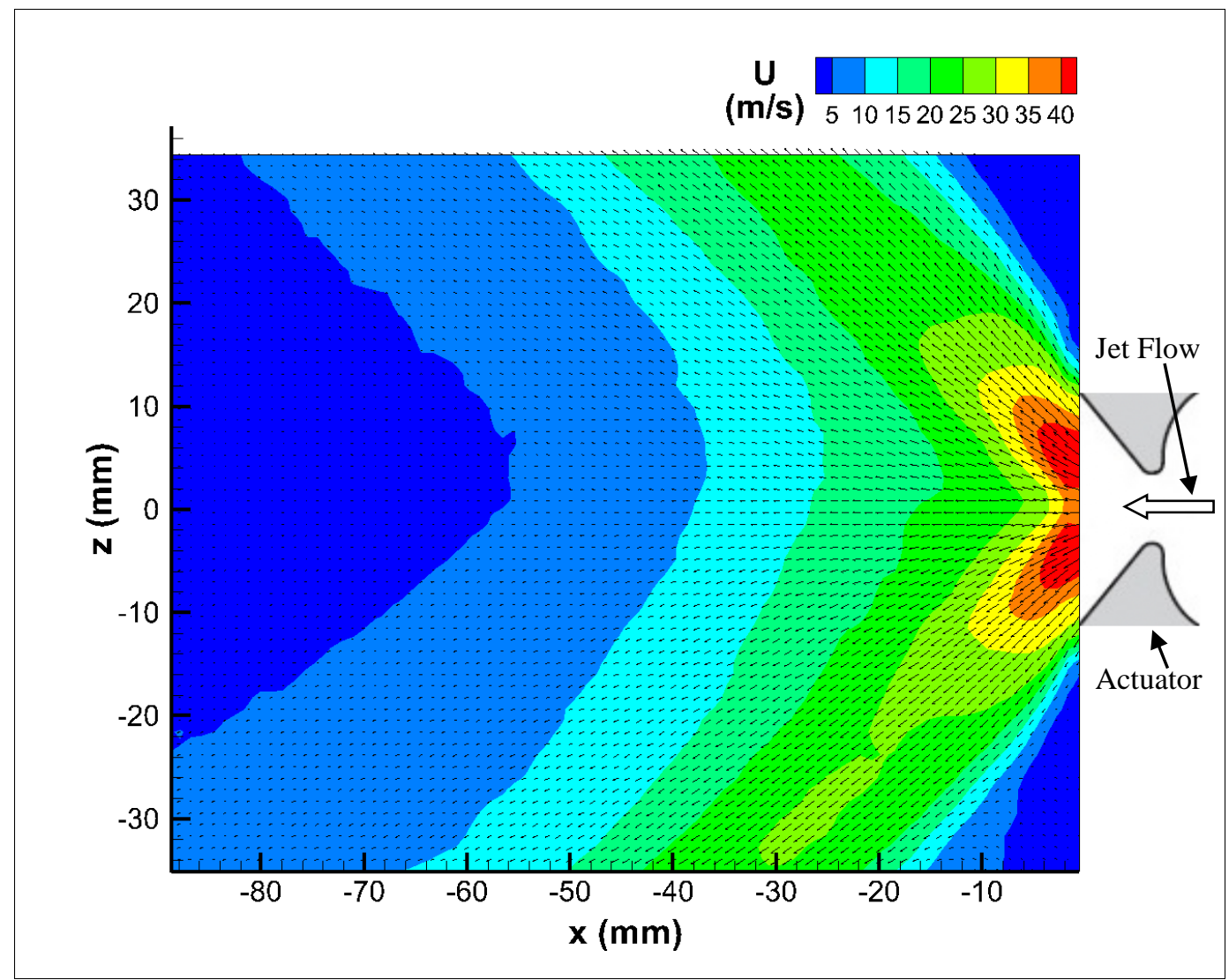

Figure 15. Time averaged velocity field for the baseline case (mass flow rate is $4.54 \mathrm{~g} / \mathrm{s}$ ).
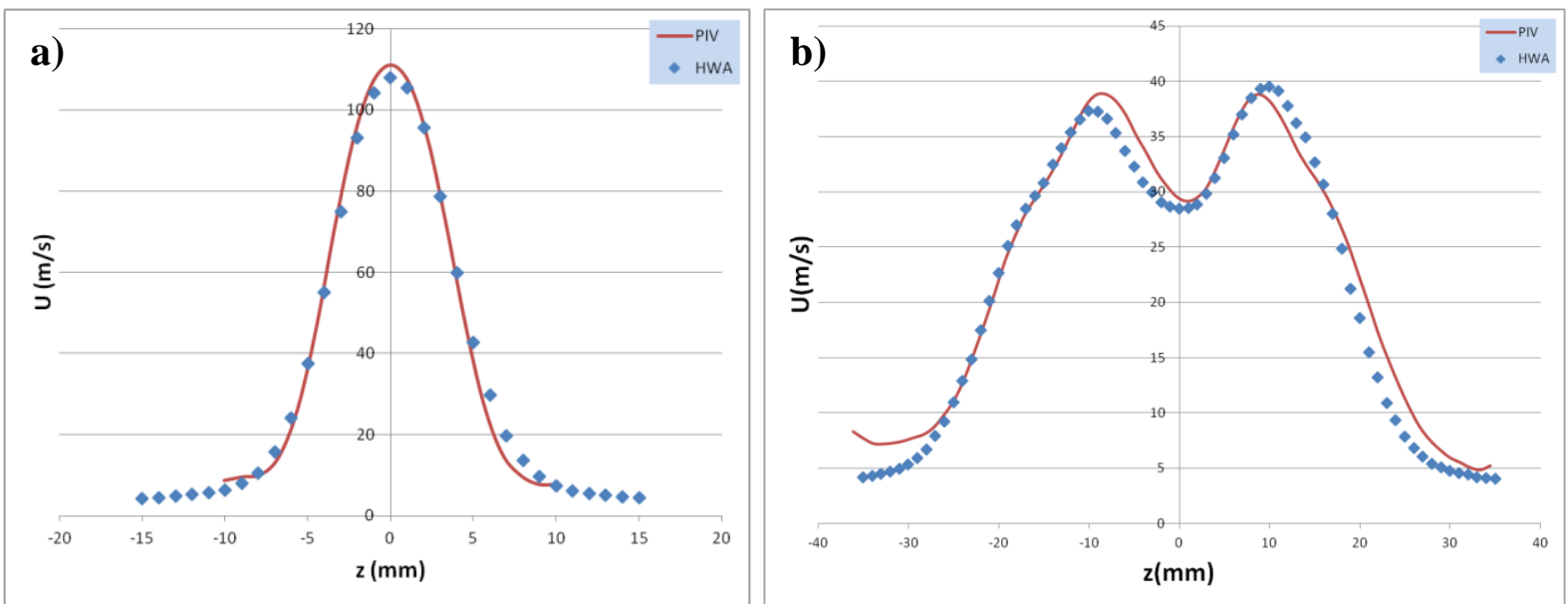

Figure 16. Comparison of PIV and HWA measurements for a) steady jet, and b) oscillating jet. The mass flow rate is $4.54 \mathrm{~g} / \mathrm{s}$ and the measurements are at $x=6 \mathrm{~mm}$ downstream location. 


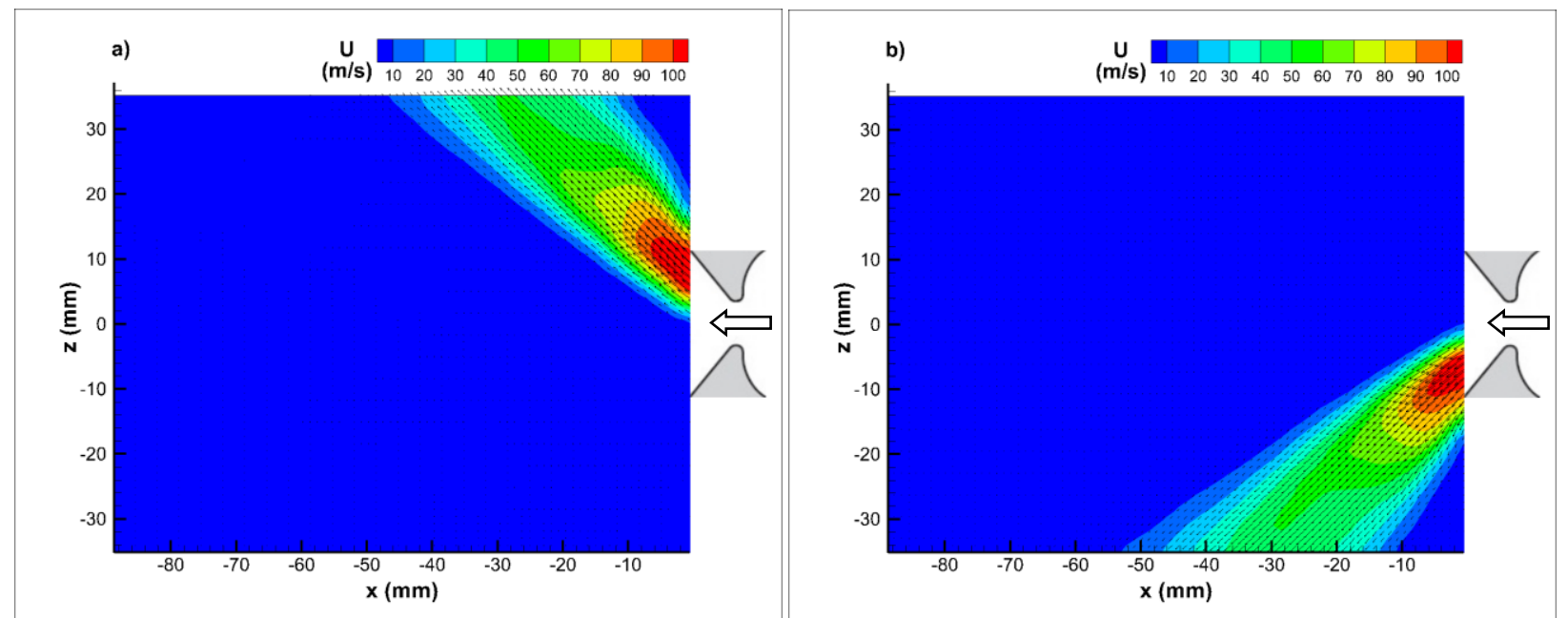

Figure 17. Non oscillating a) upward b) downward jets with modified actuator (mass flow rate is $4.54 \mathrm{~g} / \mathrm{s}$ ).

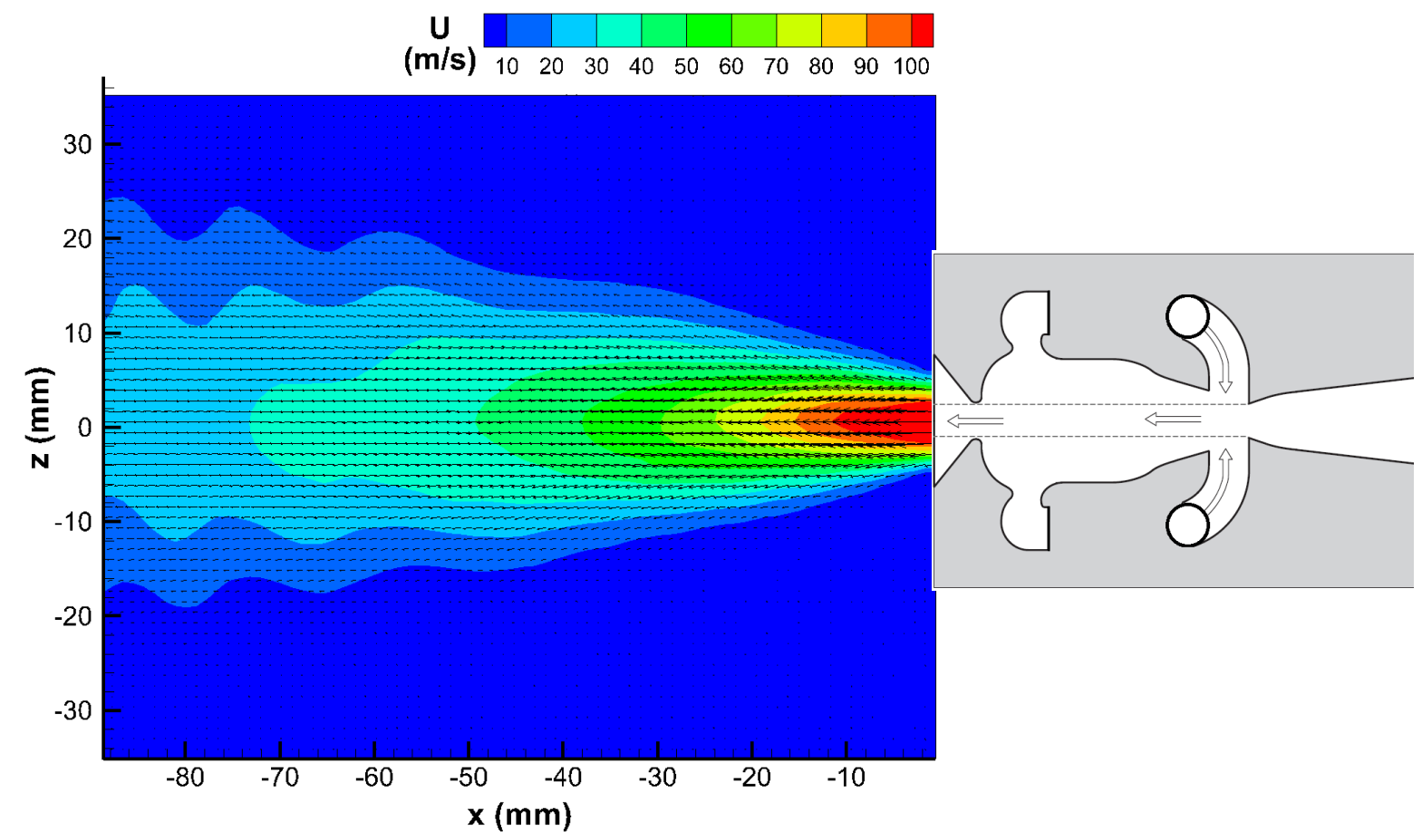

Figure 18. Non oscillating planar jet with both valves open (mass flow rate is $4.54 \mathrm{~g} / \mathrm{s}$ ). 

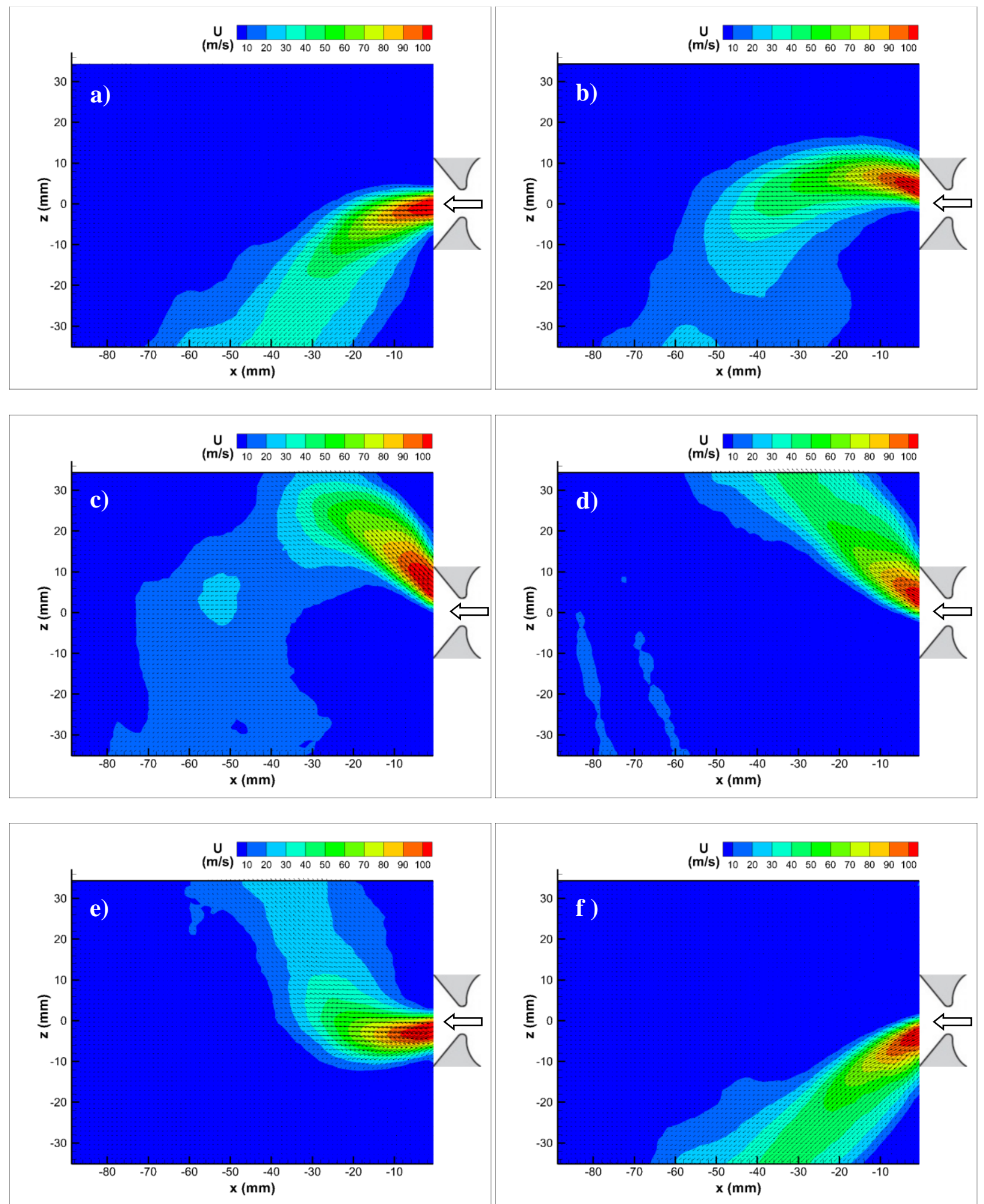

Figure 19. Evolution of actuator flow field at different phases: a) $0^{\circ}$, b) $40^{\circ}$, c) $80^{\circ}$, d) $160^{\circ}$, e) $200^{\circ}$, f) $340^{\circ}$.The mass flow rate is $4.54 \mathrm{~g} / \mathrm{s}$ and the oscillation frequency is $105 \mathrm{~Hz}$. 